\title{
HUBBLE SPACE TELESCOPE OBSERVATIONS OF THE DISK AND JET OF HH 30
}

\author{
Christopher J. Burrows, ${ }^{1,2}$ Karl R. Stapelfeldt, ${ }^{3}$ Alan M. Watson, ${ }^{4}$ John E. Krist, ${ }^{1}$ \\ Gilda E. Ballester, ${ }^{5}$ John T. Clarke, ${ }^{5}$ David Crisp,${ }^{3}$ John S. Gallagher III, ${ }^{6}$ \\ Richard E. Griffiths, ${ }^{7}$ J. JeFF Hester, ${ }^{8}$ John G. Hoessel, ${ }^{6}$ Jon A. HoltzMan, ${ }^{4}$ \\ Jeremy R. Mould, ${ }^{9}$ Paul A. Scowen, ${ }^{8}$ John T. Trauger, ${ }^{3}$ AND \\ JAMES A. WESTPHAL ${ }^{10}$ \\ Received 1996 April 25 ; accepted 1996 June 26
}

\begin{abstract}
HH 30 in Taurus has been imaged with the Hubble Space Telescope WFPC2. The images show in reflected light a flared disk with a radius of about 250 AU that obscures the protostar. The disk resembles detailed accretion disk models that constrain its density distribution and show that its inclination is less than $10^{\circ}$. There are bipolar emission-line jets perpendicular to the disk, a very clear demonstration of the standard paradigm for accretion disk and jet systems. However, asymmetries in the light distribution show that the disk has not completely settled into a quasi-equilibrium accretion state, or that some of the observed scattering is from an asymmetric envelope. The emission-line jet itself is resolved into a number of knots with typical lengths and separations of 0".4, much smaller and more numerous than indicated by lower resolution ground-based studies. There are indications of still finer structures in the jet all the way to the resolution limit of 0 ." 1 . The knots have proper motions ranging from 100 to 300 $\mathrm{km} \mathrm{s}^{-1}$ and are therefore generated at the surprisingly high rate of about 0.4 knots per jet per year. The jet appears to be collimated within a cone of opening angle $3^{\circ}$ and can be seen to within $30 \mathrm{AU}$ of the star.

Both single- and multiple-scattering disk models have a range of possible solutions, but by requiring pressure support and temperature equilibrium, a self-consistent model emerges. There is evidence for pressure support because the disk appears to have a Gaussian height profile. The temperature at each point in the disk is determined by the disk geometry, which in turn fixes the temperature in a selfconsistent manner. The extinction to the protostar is unknown but constrained to be greater than 24 mag. The optical properties of the scattering grains in the disk are determined and found to imply a large scattering asymmetry, but they seem to follow the interstellar reddening law. The absolute magnitude and colors of the unseen protostar, which has a brightness in the $I$ bandpass of about 0.16 times solar and is very red, are obtained. The disk mass is about 0.006 times solar and has an expected lifetime of about $10^{5} \mathrm{yr}$.
\end{abstract}

Subject headings: accretion, accretion disks - circumstellar matter — dust, extinction -

ISM: jets and outflows - stars: individual (HH 30) — stars: pre-main-sequence

\section{INTRODUCTION}

Herbig-Haro 30 is a nebulous low-mass star located $90^{\prime \prime}$ south of HL Tau in the Taurus molecular cloud. Historically this system was cataloged as an $\mathrm{HH}$ object on the basis of its strong [S II], [O I], and $\mathrm{H} \alpha$ emission lines. It is actually a composite system that includes a young stellar object and its associated reflection and emission nebulosities. Early CCD imaging (Mundt \& Fried 1983) revealed that the emission lines are formed in a highly collimated jet at a position angle of $30^{\circ}$. Subsequent studies (Mundt, Brugel, \& Buhrke 1987; Mundt et al. 1990) show that the jet can be traced for a full $2^{\prime}(0.1 \mathrm{pc})$ to the north-northeast and

${ }^{1}$ Space Telescope Science Institute, 3700 San Martin Drive, Baltimore, MD 21218; burrows@stsci.edu; krist@stsci.edu.

${ }^{2}$ Astrophysics Division, Space Science Department, European Space Agency.

${ }^{3}$ Jet Propulsion Laboratory, Mail Stop 183-900, 4800 Oak Grove Drive, Pasadena, CA 91109; krs@bb2.jpl.nasa.gov; dc@crispy.jpl.nasa.gov; jtt@bb4.jpl.nasa.gov.

${ }_{4}$ Department of Astronomy, New Mexico State University, Las Cruces, NM 88003; alan@oldp.nmsu.edu; holtz@mmsu.edu.

${ }^{5}$ Department of Atmospheric and Oceanic Sciences, University of Michigan, 2455 Hayward, Ann Arbor, MI 48109; clark, gilda@sunshine.sprl.umich.edu. that an oppositely directed counterjet can be traced more than $1^{\prime}$ to the south-southeast. Lopez et al. (1995) have identified additional emission knots that they associate with the north-northeast HH 30 outflow up to $5^{\prime}$ from the star, and Graham \& Heyer (1990) report that the southsouthwest outflow extends for 3'. HH 30, HL Tau, XZ Tau, and $\mathrm{LkH} \alpha 358$ are a compact association of young stellar objects (YSOs) most probably with a similar age (Mundt et al. 1987) of about $10^{5} \mathrm{yr}$ (Stapelfeldt et al. 1995). The first three objects are going through the stage of mass outflow, through highly collimated jets, that produces $\mathrm{HH}$ objects. The distance to these objects is approximately $140 \mathrm{pc}$ (Elias 1978).

${ }^{6}$ Department of Astronomy, University of Wisconsin, Madison, 475 N. Charter Street, Madison, WI 53706; jsg@jayg.astro.wisc.edu, hoessel@jth.astro.wisc.edu.

${ }^{7}$ Department of Astronomy, Johns Hopkins University, $3400 \mathrm{~N}$. Charles Street, Baltimore, MD 21218; griffth@mds.pha.jhu.edu.

${ }^{8}$ Department of Physics and Astronomy, Arizona State University, Tyler Mall, Temple, AZ 85287; jjh@cygloop.la.asu.edu; scowen@tycho.la.asu.edu.

${ }^{9}$ Mount Stromlo and Siding Springs Observatories, Australian National University, Weston Creek Post Office, ACT 2611, Australia; jrm@mso.anu.edu.au.

${ }^{10}$ Division of Geological and Planetary Sciences, California Institute of Technology, Pasadena, CA 91125;jaw@soll.gps.caltech.edu. 
Optical spectroscopy shows that the HH 30 jet and counterjet have very similar radial velocities (Cohen \& Jones 1987; Mundt et al. 1987, 1990), and thus the outflow is directed very nearly in the plane of the sky. Proper motions measured by Mundt et al. (1990) correspond to velocities of $170 \mathrm{~km} \mathrm{~s}^{-1}$, a typical flow (or pattern) speed for knots in Herbig-Haro jets. Broadband ground-based photometry shows that the unresolved central continuum component of HH 30 is quite faint $(V=19.5, I=17.3$ [Mundt \& Fried 1983]; $K=13.2$ [Vrba, Rydgren, \& Zak 1985]), which leads to a luminosity of only $6 \times 10^{-4} L_{\odot}$ (Mundt et al. 1987). No reddening estimate for the star is available.

HH 30 does not appear in the IRAS Point Source Catalog, perhaps due to confusion with nearby $\mathrm{HL}$ and $\mathrm{XZ}$ Tau. Cohen \& Schwartz (1987) have derived 12 and $25 \mu \mathrm{m}$ IRAS fluxes from the survey data of 0.23 and $0.62 \mathrm{Jy}$, respectively, and a luminosity of $0.15 L_{\odot}$. These authors' ADDSCAN profiles show that at the nominal coordinates for HH 30 IRS, there appears an extended wing in the profiles of the much brighter nearby sources, making the extraction of the HH 30 IRS contribution difficult. We have averaged the IRAS scans with the XSCANPI software and do not see a corresponding source, so perhaps these values should be regarded as upper bounds.

The integrated continuum polarization is $2.8 \% \pm 0.8 \%$ in a bandpass that extends from 4600-7000 $\AA$ (Cohen \& Schmidt 1991) at position angle $93^{\circ} \pm 10^{\circ}$. These authors suggested that the illuminating source is HL Tau; however, the subsequent discovery of the oppositely directed emission-line jets at P.A. $=30$ from $\mathrm{HH} 30$ made this hypothesis unlikely and unnecessary.

Millimeter continuum emission from circumstellar dust has been detected for HH 30 by Reipurth et al. (1993). At $1.3 \mathrm{~mm}$, the detected flux density is $35 \pm 6 \mathrm{mJy}$. This flux can be used to derive a mass of circumstellar material if the dust temperature and opacity are known. Assuming that the dust temperature is similar to that found for other $\mathrm{T}$ Tauri stars $(40 \mathrm{~K})$ and a $\beta=1$ dust emissivity law, the observed flux density corresponds to a circumstellar mass of $3 \times 10^{-3} M_{\odot}$.

An upper limit of $0.04 \mathrm{mJy}$ has been obtained at $3.6 \mathrm{~cm}$ at the VLA (Rodriguez 1995), well above the thermal continuum flux extrapolated from the $1.3 \mathrm{~mm}$ data for any reasonable dust emissivity assumptions.

\section{HST OBSERVATIONS}

HST Wide Field Planetary Camera 2 (WFPC2) observations were obtained through $\mathrm{F} 555 \mathrm{~W}, \mathrm{~F} 675 \mathrm{~W}$, and F814W at low signal levels on 1994 February 25 at a CCD operating temperature of $-76.2 \mathrm{C}$ and gain 15 . Images through F675W (gain 7) and F814W (gain 15) at a temperature of $-88.3 \mathrm{C}$ with longer exposure times were taken on 1995 January 5. The filters are a wide Johnson $V$ and
Kron-Cousins $R$ and $I$ analogs, respectively. The F675W bandpass includes several prominent nebular emission lines. In all cases HL Tau was the primary target centered on the Planetary Camera (Stapelfeldt et al. 1995), and HH 30 was imaged on Wide Field Camera 2 at a resolution of 0.1 (14 AU) per pixel.

The data were subjected to a standard reduction process primarily to remove bias, subtract dark current, and correct for flat field variations. The data obtained are summarized in Table 1, together with some photometric results that are discussed further below.

HH 30 and its surrounding nebulosity are shown in Figure 1 (Plate 9). The image shows an emission-line jet that propagates the full height of the figure in both directions from the bright continuum reflection nebulosity near the bottom.

The emission-line jet and continuum reflection nebula are also shown in the deconvolved image in Figure 2 (Plate 10), which shows that the reflection nebula separates cleanly into two components, and that much of the light seen between them in Figure 1 is due to the logarithmic stretch of that figure pulling up low surface brightness point-spread function (PSF) wings from the bright nebula. In addition, Figure 2 shows that the jet propagates all the way through both sides of the reflection nebula and disappears into the same dark lane that separates the nebulae. Unlike other bipolar nebulae, the greatest extent of the reflection nebula is in the direction perpendicular to the jet axis rather than being aligned with the jet.

The north-northeast nebula has a flattened shield or lens shape, with an apparent width of $450 \mathrm{AU}$ and maximum height of about $100 \mathrm{AU}$ at its center, where the jet crosses it. The lower edge of the nebula is smoothly curved, concave toward the jet. On the south-southwest side of the star, there is a second reflection nebula that is very roughly symmetrical about the dark lane with the north-northeast nebula. The south-southwest nebula extends to greater distances from the source than any point on the northnortheast side. At first sight, both nebulae are mirror symmetric across the jet. However, definite asymmetries exist. In Figure 2, the top right of the north-northeast nebula (farthest from the dark lane) is distinctly brighter than the top left. In the bottom left of the figure, a faint finger-like extension to the limb of the south-southwest nebula can be traced up to 3".5 from the star. The corresponding extension on the other side of the south-southwest nebula is much shorter.

The photometry given in Table 1 is based on the reduction and calibration given in Burrows (1995) and Holtzman et al. (1995). The uncertainty in the calibration is much less than the quoted errors in the table, as is the photon statistical error. Two independent observers derived the photometry in three different ways from rectangular

TABLE 1

OBSERVATION SUMMARY

\begin{tabular}{cccccc}
\hline \hline $\begin{array}{c}\text { Database } \\
\text { Identifier }\end{array}$ & Date & Filter & $\begin{array}{c}\text { Exposure } \\
(\mathrm{s})\end{array}$ & $\begin{array}{c}\text { North Nebula } \\
(\mathrm{mag})\end{array}$ & $\begin{array}{c}\text { South Nebula } \\
(\mathrm{mag})\end{array}$ \\
\hline $\mathrm{u} 26 \mathrm{~s} 0401 \mathrm{t} \ldots .$. & 1994 Feb 25 & F555W & 350 & $18.45 \pm 0.06$ & $21.11 \pm 0.18$ \\
$\mathrm{u} 26 \mathrm{~s} 0402 \mathrm{t} \ldots \ldots$ & 1994 Feb 25 & F675W & 120 & $17.16 \pm 0.05$ & $19.89 \pm 0.21$ \\
$\mathrm{u} 26 \mathrm{~s} 0403 \mathrm{t} \ldots \ldots$. & 1994 Feb 25 & F814W & 60 & $16.62 \pm 0.05$ & $19.37 \pm 0.13$ \\
$\mathrm{u} 2 \mathrm{ky0101t} \ldots . .$. & 1995 Jan 5 & F675W & 800 & $17.66 \pm 0.07$ & $19.40 \pm 0.12$ \\
$\mathrm{u} 2 \mathrm{ky0104t.....}$ & 1995 Jan 5 & F814W & 1200 & $17.08 \pm 0.06$ & $18.56 \pm 0.10$ \\
\hline
\end{tabular}


boxes centered on the nebulae. The average result is the quoted value, while the half-range is the quoted error. The errors are large because it is hard to define the precise extent of the nebulae. The photometry includes some contamination from the direct jet emission where it propagates through the nebula, particularly in $\mathrm{F} 675 \mathrm{~W}$, although this is a small fraction of the total and is impossible to separate unambiguously. The overall color of the north-northeast nebula is $\mathrm{F} 814 \mathrm{~W}-\mathrm{F} 675 \mathrm{~W}=-0.58 \pm 0.13$. An independent measurement of this color was made, masking the jet region entirely, and it gave a color difference of 0.8 .

It is clear that the nebulae changed in brightness between the observation epochs. The north-northeast nebula became fainter by $0.50 \pm 0.09 \mathrm{mag}$ in $\mathrm{F} 675 \mathrm{~W}$ and $0.46 \pm 0.07$ in $\mathrm{F} 814 \mathrm{~W}$, while the south-southwest nebula appears to have brightened by $0.49 \pm 0.25 \mathrm{mag}$ in $\mathrm{F} 675 \mathrm{~W}$ and $0.81 \pm 0.17 \mathrm{mag}$ in $\mathrm{F} 814 \mathrm{~W}$ (adding the errors in quadrature). The color-independent average changes are $0.48 \pm 0.06(\mathrm{NNE})$ and $-0.65 \pm 0.15$ (SSW). The color change of $0.30 \pm 0.32$ is consistent with zero within the noise of the observations. The brightness variations must be due to changes near the central source (together with some contribution of direct and scattering light from the time variable jet) because the timescale for changes in the distribution of the illuminated material is much longer than the year between the observations. If the central source becomes hotter and therefore brighter or the extinction near it decreases, the reflection nebulae would brighten and appear bluer. Some of the brightness changes should also be attributed to reflected and contaminating time-variable line emission from the jet near the central source (which appears predominantly in $\mathrm{F} 675 \mathrm{~W}$ ). These two mechanisms would also tend to make F814W - F675W color increase when the nebula brightens.

Figure 3 shows photometric contour plots of all the data. The spatial and temporal brightness and color variations across the nebula that are given in Table 1 can be seen in this figure. The similarity of these contours to the predicted contours in Figure $6 a$ of Whitney \& Hartmann (1992) leads us to identify the dark lane as caused by extinction in the accretion disk that surrounds the protostar, and the continuum nebulosity as caused by reflection of light generated near the central object. The reflection is occurring near the upper and lower surfaces of the flared and optically thick disk. The central protostar is not visible because it is heavily obscured by the disk, which also explains the low bolometric luminosity of the object. We will show detailed model fits to support this interpretation.

The more extended nebulosity visible from $8^{\prime \prime}$ to $13^{\prime \prime}$ above HH 30 in Figure 1 is seen most clearly in F814W, where it has a peak surface brightness of $22.0 \mathrm{mag} \mathrm{arcsec}^{-2}$. It is fainter but still visible in F675W. Its F675W - F814W color is $0.3 \mathrm{mag}$, so it is almost certainly continuum radiation, as emission-line sources are in general much brighter in F675W. It is only visible in a region that is accessible to direct illumination from the central object, without significant extinction from the circumstellar disk. Therefore, it is probable that it is scattered light from the central object. This conclusion can be checked with polarization measurements. This reflected light gives us an opportunity to study the central object more directly, but the signal-to-noise ratio and spectral coverage of the present data set prevent this. Scattering will tend to be more efficient in the shorter wavelength passband, but even so, the color of this nebula

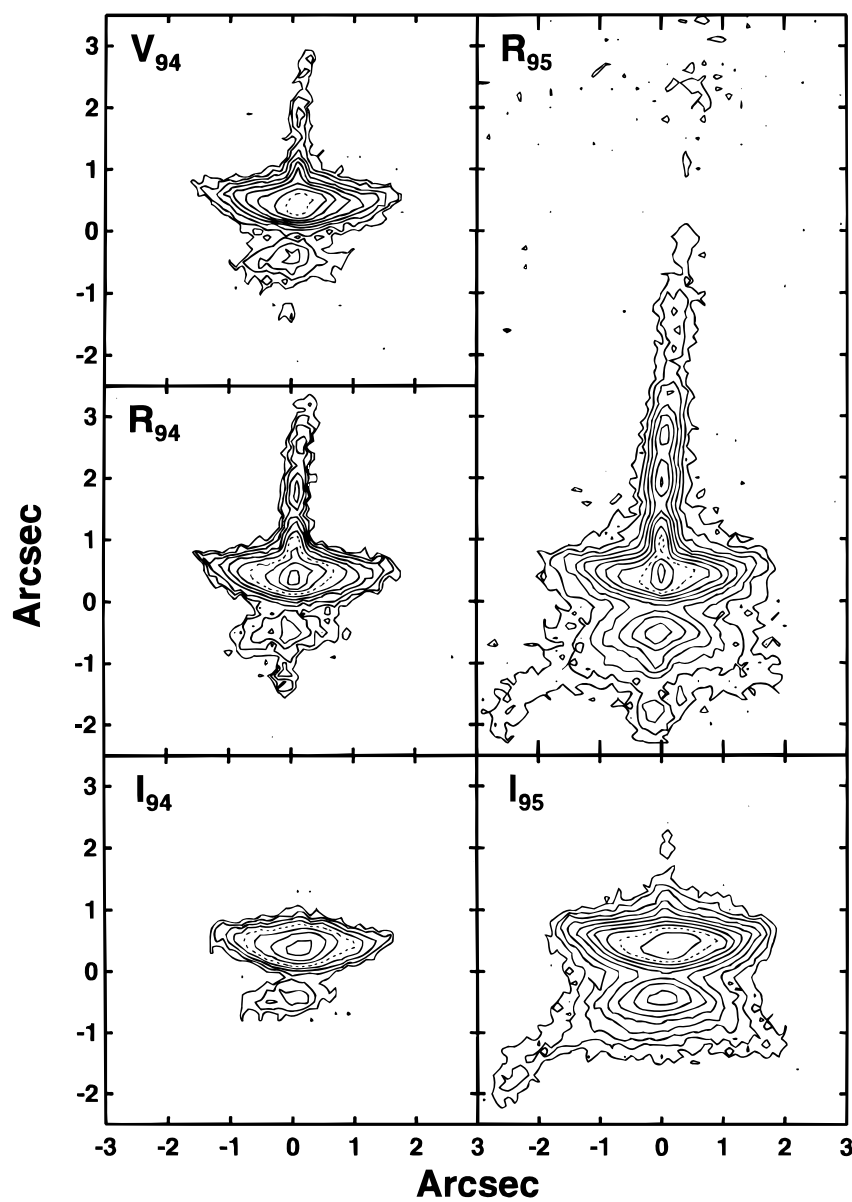

Fig. 3.-Contour plots of all the data. The dotted contour is at a level of 18 th magnitude per square arcsecond. The contours are spaced by 0.5 mag. For the 1994 data, position angle 35.4 is up, while for the 1995 data, P.A. $=34$ is up.

clearly precludes heavy reddening. This nebula is significantly bluer than the $\mathrm{HH} 30$ reflection nebula itself, which has an F675W - F814W color of $0.8 \mathrm{mag}$, when the jet region is excluded. The brightest region of the more extended nebula is discussed specifically in Mundt et al. (1990). They too conclude that it is a reflection nebula, and they speculate that it may be illuminated by an additional source, but we see no evidence for such a source.

\section{THE EMISSION-LINE JETS}

The jet is very bright in the F675W filter, which includes the brightest $\mathrm{HH}$ object emission lines ([S II $] \lambda \lambda 6717,6731$, $\mathrm{H} \alpha 26563$, and [O I] 26300). Little emission from the jet is seen in the F814W filter, which does not include any bright $\mathrm{HH}$ object emission lines. The jet consists of discrete resolved knots that appear to move away from the star between the two observation epochs.

Figure 4 (Plate 11) has been constructed in order to allow study of the jet morphology and proper motions over the entire detected lengths. The jets decrease rapidly in intensity away from the protostar. For display clarity, the intensity of the image was therefore changed by multiplying each pixel by the square of its distance from the protostar, after subtracting a constant background. To the north-northeast, the jet extends for at least $2^{\prime}$, but it becomes invisible in our data after about 16" in 1995 and $5^{\prime \prime}$ in 1994 because of signal-tonoise ratio limitations. To the south-southwest, all the data 
are incomplete because the counterjet is truncated by the edge of the CCD frame. The protostar position is marked with a cross and is based on the disk models discussed in the next section.

At least eight bright knots can be seen within $5^{\prime \prime}$ of the source position. At least an additional six knots can be seen between $5^{\prime \prime}$ and $16^{\prime \prime}$ in the 1995 data. The jet is typically very narrow, with a FWHM of only about 0 ".15 (20 AU) near the source, and it can be traced easily to within 0.2 of the stellar position. Two emission knots are seen south-southwest of the star, exactly on the axis of the bright jet. These jet components are labeled so as not to conflict with the nomenclature adopted by Lopez et al. (1995), who extended that adopted by Mundt et al. (1990). At this resolution, it is appropriate to label the knots with the year of observation, to allow for future mergers, for new knots to appear, and for new knots to be discovered at higher resolution or signalto-noise ratio.

In the right panel of Figure 4 the outer region of the detected jet is shown. It can also be discerned in Figure 1. The jet is not straight. It bends towards the north by about $5^{\circ}$ near knot $95-04 \mathrm{~N}$, reaches a second maximum deviation from its average direction at $95-10 \mathrm{~N}$, and then crosses back to a third extremum between $95-13 \mathrm{~N}$ and $95-14 \mathrm{~N}$. This behavior is consistent with the jet axis precessing, but other explanations are also quite possible at this stage. We have fitted by eye the expected geometric curve that a precessing jet would present in projection through the observed jet knots, and this curve is overlain in the right panel of Figure 4. The parameters correspond to the average jet axis circling a cone centered at position angle $30^{\circ} .8$. The cone has a half-opening angle of $2: 4$. The jet would have a precession period of 42 years if we assume an average stream velocity of $200 \mathrm{~km} \mathrm{~s}^{-1}$. However, these direction changes may instead be caused by variations in the density of the external medium if the jet is coupled to it. In particular, the first bend is near the start of the faint reflection nebula to the northnortheast of $\mathrm{HH} 30$. It should be possible to distinguish between these two possibilities over the next few years by direct measurements of the transverse velocities of the jet with HST. The knots will either tend to continue along the existing curve or have peak transverse velocities relative to it of order $8 \mathrm{~km} \mathrm{~s}^{-1}$, if the motion at each point is purely radial.

We have started to study the knot motions by careful astrometry on the existing images. The reduced images were registered by first translating the 1995 data so that the reflection nebulae aligned. Next the image was rotated by 1.4 (the difference in the known spacecraft position angle), and finally it was rescaled by a factor of 0.994 to account for differences in the local scale on the CCD caused by optical distortion. The coordinates of each knot at each epoch were then determined by drawing identical sized boxes and registering the boxes relative to the knot by blinking the resulting subimages. This gave the knot offset to an accuracy of a pixel. An additional correction accurate to a fraction of a pixel was then obtained by computing the centroid of the knots within their respective boxes. This procedure gives the positions and velocities shown in Table 2. The position of the star was determined from the best disk models (see below) and defines the origin of the coordinate system. The orientation of the coordinate system for the table was chosen to minimize the rms scatter in the $x$ coordinate, excluding 95-6 and 95-7, which, as noted above,
TABLE 2

Observed KNot COORdinates AND Proper Motions

\begin{tabular}{|c|c|c|c|c|}
\hline \multirow[b]{2}{*}{ KNOT } & \multicolumn{2}{|c|}{ Position (AU) } & \multicolumn{2}{|c|}{ VELOCITY $\left(\mathrm{km} \mathrm{s}^{-1}\right)$} \\
\hline & $x$ & $y$ & $x$ & $y$ \\
\hline $95-02 \mathrm{~S}$ & -0.7 & -249.6 & 27 & -279 \\
\hline $95-01 \mathrm{~S} \ldots$ & -0.2 & -132.2 & 16 & -227 \\
\hline $95-01 \mathrm{~N}$ & 1.6 & 71.8 & 1 & 54 \\
\hline $95-02 \mathrm{~N} \ldots \ldots \ldots$ & 2.9 & 159.9 & -20 & 258 \\
\hline $95-03 \mathrm{~N} \ldots \ldots \ldots$ & -3.5 & 281.0 & 16 & 158 \\
\hline $95-04 \mathrm{~N} \ldots$ & -2.1 & 382.0 & 12 & 84 \\
\hline $95-05 \mathrm{~N} \ldots \ldots \ldots$ & 2.1 & 469.0 & -18 & 134 \\
\hline $95-6+7 N \ldots \ldots$ & 10.9 & 635.4 & 10 & 166 \\
\hline
\end{tabular}

are not collinear with the others. The $y$-axis is therefore defined to be the jet axis nearest the star for which we derive a position angle of 31.3 .

The errors in this analysis can be estimated by assuming a centroid error of 0.2 pixels, which is reasonable in view of the detector's undersampling and the low signal-to-noise ratio of this data. This centroid error corresponds to a position error of $3 \mathrm{AU}$, which is comparable to the spread in $x$ positions seen in the table, and a velocity error of $22 \mathrm{~km} \mathrm{~s}^{-1}$ (when the fact that two centroids contribute to the velocity uncertainty is taken into account).

From the table, we can see that the knots on both sides of the disk are arranged roughly in a line through the star perpendicular to the disk. They propagate away from the star along this line. The longitudinal velocities vary significantly between 100 and $300 \mathrm{~km} \mathrm{~s}^{-1}$ from knot to knot. Therefore, if each knot represents a material concentration in the jet, mergers and collisions downstream can be expected. From the preponderance of $[\mathrm{S}$ II $]$ in the jet spectrum, these collisions, if they occur, must result in relatively low ionization shocks with velocity gradients of $30-50 \mathrm{~km}$ $\mathrm{s}^{-1}$. The velocity differences between the knots would have to be accounted for in a number of shocks. Alternatively, the knots could represent moving shock patterns in a flow that has much less longitudinal velocity structure than the proper motions imply. Again, a detailed study of the temporal evolution of the jet should enable us to distinguish between these possibilities.

The transverse velocities are consistent with zero but are also consistent with the jet motion being helical.

The knots are not equally spaced but have spacings ranging from 90 to $120 \mathrm{AU}$. The knot ejection rate is therefore of order one knot every $2.5 \mathrm{yr}$. At lower resolution, the same characteristic velocities can be seen, but about 7 times fewer knots, so one would have inferred that ejection events occur every 20 years or so. We see now that they are much more frequent.

There is not a one-to-one correspondence between the knots in the jet and the counterjet. Therefore, if the knots are caused by discrete accretion events from instabilities in the inner accretion disk, the events are not symmetric and tend to eject material into one jet or the other, or the knots evolve and accelerate differently in the jet and counterjet.

In addition, we investigated the width and brightness of the jets as a function of the distance from the star. This was done best with the deconvolved image shown at the top of Figure 5 (Plate 12), although similar conclusions can be drawn from the raw image. The maximum entropy deconvolved image was obtained with a TINYTIM point-spread function (PSF) (Krist 1995). A series of cuts were made 
through the deconvolved image at various distances from the star. The width of the jet at each point was defined by fitting a Gaussian with variable width (to represent the jet) plus a variable flat background to roughtly represent the continuum reflection nebulosity. The result of this fit is also shown in Figure 5. The width of a deconvolved unresolved point source was found to be equal to the width of the jet measured close to the star. The FWHM of the jet is less than $20 \mathrm{AU}$ at $70 \mathrm{AU}$ from the star (and probably much less) and appears to grow linearly away from the star with a FWHM-to-distance ratio of 1:20, which corresponds to a cone opening angle of $3^{\circ}$ between 0.5 and $5^{\prime \prime}$ from the star. The counterjet appears to have a wider opening angle that is perhaps twice as large, a result also reported in Mundt et al. (1990) at larger distances. These authors also show that the jet opening angle is almost constant at 1.9 from $2 . .5$ to $75^{\prime \prime}$ from the star. Therefore, the jet appears to recollimate. We appreciate that the emission-weighted jet width may not be an accurate representation of the mass-weighted width; however, any model of jet propagation will need to produce these qualitative features in emission measure.

The jet decreases rapidly in brightness as the distance $d$ from the star increases. The bottom panel of Figure 5 shows that the average integrated brightness per unit length falls roughly as $d^{2}$. The knots may be excited by internal shocks within the jet, but this is not necessary. At $2^{\prime \prime}$ along the north-northeast jet, Mundt et al. (1990) estimate an electron density of $700 \mathrm{~cm}^{-3}$, and an even smaller value further out. This corresponds to a recombination time of 160 years, which is long compared to the 20 years or so it takes for a knot to propagate the 6 " shown in the figure. If the knots are ionized individually close to the protostar, and then freely and linearly expand transversely (as indicated by the linear jet expansion), the brightness variation is understandable and no additional energy input is necessary. In this case, the emission per unit volume will depend on the number density squared, which in turn falls as $d^{2}$. The crosssectional area of the jet increases as $d^{2}$, so the overall emission per unit length would decrease as $d^{-2}$ as observed. If the knots were to expand longitudinally as well as transversely, the average brightness would fall as $d^{4}$, which is inconsistent with the observations. Therefore, if no additional ionization is occurring close to the star (presumably before the jet couples to the external medium), the jet knots must be in longitudinal pressure equilibrium with neutral or more tenuous material.

\section{THE REFLECTION NEBULOSITY}

The 1995 January F814W image shows the reflection nebulosity most clearly, as it does not include the many strong nebular emission lines present in the F675W image, and yet it has a much higher signal-to-noise ratio than any of the 1994 February images. The reflection nebula extends perpendicular to the jet close to the jet's base. It has two components: a brighter one to the north-northeast of the star, and a fainter one to the south-southwest. The projected position of the protostar itself is inferred from the nebular models discussed below to be on the jet axis, between the reflection nebulae and somewhat closer to the edge of the north-northeast nebula. The protostellar photosphere is not seen directly because of extinction in the circumstellar nebula.

The observed morphology of the two reflection nebulae is strikingly similar to models of an optically thick circumstel- lar disk viewed from near the equatorial plane. The similarity is compelling because of the dark lane of obscuration that separates the two reflection nebulae, its nearly perfect symmetry, and increasing width of the dark lane with radial distance from the star. Starlight does not penetrate the interior of an optically thick disk; instead it illuminates only the upper and lower surfaces of the disk. Whitney \& Hartmann $(1992,1993)$ have calculated models of such disks and found that the reflected light is very difficult to observe unless the disk obscures the direct light of the central star. With the assumption that the disk is perpendicular to the jets, and the fact that the jets from $\mathrm{HH} 30$ are known to be flowing close to the plane of the sky, we expect that the HH 30 disk is being viewed edge-on and thus will obscure the central star.

Using an aperture that excludes line emission from the bright jet, we measure an integrated $R-I$ color of $0.8 \mathrm{mag}$ nitudes for the scattered starlight. An unreddened late-type photosphere has an intrinsic $R-I$ color of $0.5 \mathrm{mag}$. Thus, the observed colors of the HH 30 nebulosity are consistent with minimal reddening for the scattered light that reaches the observer. This argues against the presence of any significant extended circumstellar envelope that would produce additional reddening that is not observed.

It is clear that the brightness changes over the year between the observations cannot be due to changes in the nebular density distribution because the dynamical timescale is of order $10^{3} \mathrm{yr}$. The observed changes must be caused by variability and anisotropy in the central light source or the distribution of material very close to it. The timescale for this variability is comparable to the emission timescale for the knots observed in the jet and must be caused by changes within 1 AU or so of the source. Despite this evidence for anisotropy, we model the central source as radiating equally to the two sides of the disk. This assumption affects mainly our estimates of the disk inclination, which are very uncertain in any case.

The polarization position angle of $93^{\circ} \pm 10^{\circ}$ (Cohen \& Schmidt 1981) is not parallel or perpendicular to the jet, as expected with a central illuminating source with a cylindrically symmetrical reflection nebula inclined to the line of sight. We believe that this is because the net polarization contains significant components due to the intrinsic asymmetries of the nebula, and the contribution from the symmetric parts of the nebula is small (see, for example, Whitney \& Hartmann 1992, Fig. 8). For example, in Figure 2, a bright asymmetric bar can be seen near the north edge of the reflection nebula.

The WFPC2 images of the HH 30 nebulosities offer an unprecedented opportunity to study the spatial structure of a young stellar object disk at high resolution. In particular, constraints on the radial variation of disk thickness can be obtained by fitting the shape of the two reflection nebulosities. It is also possible to constrain better the intrinsic luminosity of the HH 30 exciting star and the reddening. We describe the model first in a parametric form, and two computer implementations, and then we outline the results obtained for each parameter in the model.

\section{MODELS FOR THE REFLECTION NEBULOSITY}

We assume axial symmetry and use simple power-law approximations for the disk surface density $\Sigma(r)=$ $\Sigma_{0}\left(r / r_{0}\right)^{\alpha+\beta}$ and scale height $H(r)=H_{0}\left(r / r_{0}\right)^{\beta}$. The mass density at any point in the disk then takes the form 
$\rho(r, \theta, z)=\rho_{0}\left(r / r_{0}\right)^{\alpha} \exp \left[-|z|^{\xi} / 2 H^{\xi}(r)\right]$, where $\rho_{0}$ is the disk midplane density at the reference radius $r_{0}$. The index $\xi$ is 1 for an exponential disk vertical profile or 2 for a Gaussian profile. As a convenient reference radius, we choose $r_{0}=100 \mathrm{AU}$. The axis of the system is inclined to the plane of the sky by an angle $l$ (which is clearly small). The disk extends from an inner cutoff $r_{\min }$ to an outer cutoff $r_{\max }$.

In the theoretically important case of a Gaussian vertical profile when $\xi$ is 2 , integration gives $\Sigma_{0}=(2 \pi)^{1 / 2} H_{0} \rho_{0}$. When $\xi$ is $1, \Sigma_{0}=4 H_{0} \rho_{0}$. In any case, the total mass in the disk is $M=2 \pi \Sigma_{0} r_{0}^{2}\left(r_{\max }^{\gamma}-r_{\min }^{\gamma}\right) /\left(\gamma r_{0}^{\gamma}\right)$, where $\gamma=\alpha+\beta+2$. The result only depends significantly on the inner or outer cutoff, depending on the sign of $\gamma$.

All the visible light is expected to be generated near the center of the disk on a scale well below HST's spatial resolution. It is a sum of thermal emission from the hot inner regions of the accretion disk and emission from the protostar itself. We approximate the sum of these two sources by a point source with a total flux $F_{\lambda}$ at effective wavelength $\lambda(=555,675$, or $814 \mathrm{~nm})$ at the origin. The light is scattered (perhaps multiply) and absorbed by the grains before some of it can escape from the nebula.

The grain properties are assumed to be independent of position in the nebula because all of our data are taken outside the sublimation radius of the probable significant ices, although particle size may evolve as a function of radius, so this assumption may be a simplification. To characterize the optical properties of the grains, we must specificy an opacity, an albedo, and a scattering phase function.

We have assumed an opacity of $\kappa=120 \mathrm{~cm}^{2} \mathrm{~g}^{-1}$. This opacity corresponds, for example, to $1 \mu \mathrm{m}$ diameter spherical particles with a density of $1.25 \mathrm{~g} \mathrm{~cm}^{-3}$ and a gas-to-dust ratio of 100 , if the scattering cross section is approximated roughly by the geometric cross section. This opacity is comparable to typical interstellar medium (ISM) values, even though the dust will probably not be well characterized by one size and the above approximation is crude. Changing the assumed opacity $\kappa$ does not affect the resulting light distribution if a compensating change is made in the fiducial density $\rho_{0}$ so that $\rho_{0} \kappa$ is unchanged. If this product is held constant, the scattering and absorption cross sections per unit length remain constant at each point in the nebula, and the distribution and intensity of light scattered by the nebula will not change. The crude mass estimates given here are therefore inversely proportional to the assumed opacity. All conclusions about the three-dimensional density scaling laws are unaffected by the assumed opacity.

We assume further an albedo of $a=0.5$. The MRN interstellar mixture (Mathis, Rumpl, \& Nordsieck, 1977) has an $I$-band albedo of 0.45 , and adding larger particles (which are absent in that mixture) will tend to increase the albedo. Witt, Oliveri, \& Schild (1990) measure the ISM grain albedo of 0.6 in $I$. On the other hand, most solar system ices and silicates have lower albedos.

We will show that a single-scattering approximation is largely adequate for characterizing the nebula. In the singlescattering approximation, what we see is unchanged if $a$ is varied but the product $a F_{\lambda}$ is kept constant. Thus, to a good approximation, uncertainties in the albedo affect only the estimated central source luminosity. Changes in the assumed albedo do affect the predicted light distribution if multiple-scattering corrections are considered, but the changes are small, and we are therefore unable to constrain the albedo. The luminosity estimates given here are inversely proportional to the assumed grain albedo.

On the other hand, we have found that the optical scattering properties of the grains in the nebula are constrained significantly by the data. We used a HenyeyGreenstein scattering phase function to describe the way in which scattered light is distributed relative to the incoming beam. This function is of the form $d \sigma / d \Omega=\sigma_{\text {tot }}\left(1-g^{2}\right) /$ $\left(1+g^{2}-2 g \cos \theta\right)$. The parameter $g=\langle\cos \theta\rangle$ defines the degree of forward scattering.

Two entirely separate numerical calculations were performed to simulate the reflection nebulosity that would be produced when the above density distribution with the above optical properties is centrally illuminated. The first was to compute an image in the single-scattering approximation. For each point in the nebula, the observed intensity is computed and projected onto the two-dimensional simulated image. The computation includes geometrical factors, the extinction to and from the scattering point, and the phase function to scatter into our line of sight. This procedure is fast, and the unknown parameters can be solved for in a least-squares manner using techniques developed to model the $\beta$ Pictoris disk (Burrows et al. 1996).

The second numerical approach is motivated by the observation that the central star is invisible in our data, and so the nebula is optically thick. Therefore, a multiplescattering approach is needed in principle. This was done with a separate Monte Carlo simulation (PINBALL) that tracks the history of many photons emitted independently from the central source, each being scattered randomly until they either escape from the nebula or are absorbed. PINBALL was originally designed to model starburst galaxies (Watson 1994), but it has since been modified to model scattering in circumstellar material. PINBALL was also modified to keep track of the number of scatters experienced by each photon, and therefore the image generated by the single scattered photons that escape from the nebula could be compared to the image generated in the independent single-scattering code. These results were also compared to analytical results in limiting cases. These tests convinced us that both the independent pieces of software are performing correctly.

As a final step in each of the two simulations, the model images were convolved with a TINYTIM PSF (Krist 1995) so that they could be compared directly to the observed data. To summarize, the model image is a function of the independent parameters $\xi, \alpha, \beta, \imath, r_{\min }, r_{\max }, H_{0}, \kappa \rho_{0}, F_{\lambda}, a$, and $g$. The parameters with subscript 0 depend implicitly on the fixed reference radius $r_{0}=100$ AU. In the singlescattering approximation, the model is a function of $a F_{\lambda}$ and not these two variables separately. For definiteness, the opacity $\kappa=120 \mathrm{~cm}^{2} \mathrm{~g}^{-1}$ and the albedo $a=0.5$ have been fixed at nominal values. To compare a model to the imaging data, $x$ and $y$ offsets and a position angle are also needed.

Important derived parameters are the fiducial surface mass density $\Sigma_{0}\left(\xi, H_{0} \rho_{0}\right)$, total disk mass $M\left(\alpha+\beta, \Sigma_{0}, r_{\min }\right.$, $\left.r_{\text {max }}\right)$, and line-of-sight extinction $A_{I}\left(\alpha, \beta, \xi, l, r_{\min }, r_{\max }, H_{0}\right.$, $\left.\kappa \rho_{0}\right)$.

We have independent constraints on some of the parameters, and these constraints can be used to guide the results. For reasons discussed below, $A_{I}$ has been constrained to be 30 mag in most of the fits. We also know by analogy with other young stellar objects (YSOs) that the source luminosity is unlikely to be greater than $2 L_{\odot}$, and the disk mass is unlikely to be many solar masses. These two constraints 
are not implemented in the fitting software, but they can be used to eliminate unlikely results. The results for each parameter and checks on our assumptions are described in the next section and placed in a theoretical context.

\section{SIMULATION RESULTS}

The highest signal-to-noise ratio data that are relatively uncontaminated by nebular emission lines are the 1995 $I$-band data. This has been used for most of the fits, except when we investigate the color dependence of the scattering parameters. The canonical best-fit disk model has $\alpha=-2.2, \quad l=7.5, g=0.64, \rho_{0}=4.1 \times 10^{-17} \mathrm{~g} \mathrm{~cm}^{-3}$, $H_{0}=15.5 \mathrm{AU}$, and $F_{I}=0.16 F_{I \odot}$. By definition, $r_{0}$ is 100 AU. For reasons that are discussed below, fixed parameters are $\xi=2, \beta=1.45, r_{\min }=0.5 \mathrm{AU}, r_{\max }=250 \mathrm{AU}$, and the extinction constraint is $A_{I}=30 \mathrm{mag}$. In addition, as discussed above the opacity $\kappa$ is $120 \mathrm{~cm}^{2} \mathrm{~g}^{-1}$, and the albedo $a$ is 0.5 . These parameters imply that $M_{\text {disk }}=0.0004 M_{\odot}$, although a more careful analysis (see below) indicates that the disk mass is about $0.006 M_{\odot}$.

Figure 6 (Plate 13) shows a comparison of this leastsquares fit to the data, and the corresponding multiplescattering simulation results. The top left panel shows the 1995 I-band image of the reflection nebula, which can be compared directly to the corresponding best-fit singlescattering approximation image in the center top panel, and the corresponding multiple-scattering simulation in the bottom left panel. One can clearly see the remarkable similarity between the HH $30 \mathrm{I}$-band image and this model.

In order to understand the uncertainties in the assumptions, models, and derived parameters, we have fixed them individually at various values and examined the models that result when other parameters are optimized to give a best fit. This set of fits is shown in Table 3, where the parameters in italics are fixed and the rest are then solved for. A numerical guide to the quality of the fit is the $\chi^{2}$ error metric. A difference of 1 in the value of $\chi^{2}$ is normally regarded as statistically significant and gives a visibly inferior fit. This quantity is minimized in the fitting process and is also given in Table 3. It shows that the canonical model fits the data within $5 \sigma$ on average. In regions of the image in which the count rate is high, the typical signal-to-noise ratio is 50 . Thus, the best-fit model reproduces the data values to an accuracy of $10 \%$ in these regions.

The canonical best fit is presented in row A1 of Table 3. Rows A2 and A3 show a very important property of the solutions. They show two local minima of the merit function connected by a line of solutions, all of which have statistically indistinguishable values of $\chi^{2}$. It is possible to fit the data with large $\beta$ and large negative $\alpha$ (A2) or by smaller $\beta$ and less negative $\alpha$ (A3). The particular solution presented in row $\mathrm{A} 1$ has been selected for physical reasons, but a wide range of other models can also fit the data.

Many of the other fits in Table 3 are visibly worse than the canonical fit given in the first row, so we can be confident that the true parameters lie in the range between such extreme constrained values and probably lie closer to the best-fit value. Several of these less satisfactory fits are shown in Figure 7 (Plate 14). In the following subsections, we examine each parameter and assumption in detail and compare to theoretical expections.

\subsection{Multiple-Scattering Correction}

The multiple-scattering code produces a single-scattering plane component and mutliple-scattering corrections that can be seen separately in Figure 6 before convolution with the instrumental PSF. The single-scattering component is (apart from statistical fluctuations) identical to the fitting software's result. This is an important validation of both implementations. The reason there is any discernible difference between the fit image and the corresponding singleplane approximation from the multiple-scattering simulation is that the former is presented convolved by the PSF for direct comparison with the data.

The integrated light from the multiple-scattering correction is $28 \%$ of the total. Although the nebula is optically thick, as can be seen from the figure, this $28 \%$ multiple scattering correction has a very similar distribution of light to the single-scattering simulation. A first scattering tends to occur where the disk material is densest near the central source. In the more distant regions of the disk, this scattered light appears to originate close to the central source, and so it produces a similar scattered radiation pattern to light that does originate there. In addition, the $n$th multiplescattering correction is suppressed by the $n$th power of the albedo. It is unlikely that the albedo is much more than our assumed value of 0.5 , so the multiple-scattering correction given here is unlikely to be significantly underestimated.

The multiple-scattering correction is most significant in the dark lane between the scattering nebulae. The extinction is so high that virtually no light reaches this region directly. Specifically, at the center of the dark lane at the $100 \mathrm{AU}$ fiducial radius the observed count rate is $11 \mathrm{DN}$. The singlescattering approximation gives $0.004 \mathrm{DN}$. When the singlescattering model is convolved with the PSF, the result is 5.5 DN, which represents the level of light scattered and diffracted in the telescope and camera from brighter regions of the nebula. The multiple nebular scattering correction at this point is $4.6 \mathrm{DN}$, which is just below the telescope background. The full multiple-scattering result convolved with the PSF gives 9.8 DN, which agrees well with the observed light level. Therefore, light must scatter at least once in order to illuminate these regions, and this is probably being observed. As the albedo is increased, the amount of light in this region will increase, and this gives in principle an albedo measurement. However, the resulting constraint is weak because the signal is below the telescope background even for an albedo of 0.5 .

In summary, the multiple-scattering correction is significant in the dark lane that separates the two halves of the HH 30 nebula, but it can be ignored for the purposes of model fitting elsewhere. In that region, multiple scattering can explain fully the difference between the single-scattering fit and the data.

\subsection{Weighting and Masking Scheme}

The detailed result that is obtained from fitting the data depends on the merit function chosen. The $\chi^{2}$ error metric was chosen to measure the quality of each fit and was minimized. In order to compute it, the variance associated with each observation point is needed. Specifically, the variance associated with a given dark and bias-subtracted pixel with $I \mathrm{DN}$ at gain $G=15$ electrons per $\mathrm{DN}$ was assumed to be the sum of contributions from the read noise $\sigma_{\text {read }}=5$ electrons rms, from flat-field variations $\sigma_{\text {flat }}=0.01$, from shot noise, and from dark current at rate $D$ for the total exposure time $T$ : 
TABLE 3

Results OF THE Model FitTing

\begin{tabular}{|c|c|c|c|c|c|c|c|c|c|c|c|c|}
\hline Run & $\xi$ & $\alpha$ & $\beta$ & $\begin{array}{c}i \\
(\operatorname{deg})\end{array}$ & $g$ & $\begin{array}{c}\rho_{0} \\
\left(\times 10^{17} \mathrm{~g} \mathrm{~cm}^{-3}\right)\end{array}$ & $\begin{array}{c}H_{0} \\
(\mathrm{AU})\end{array}$ & $F_{I} / F_{\odot}$ & $\begin{array}{c}A_{I} \\
\text { (mag) }\end{array}$ & $\begin{array}{l}M / M_{\odot} \\
\left(\times 10^{4}\right)\end{array}$ & $\chi^{2} / n$ & Notes \\
\hline $\mathrm{A} 1 \ldots \ldots$ & 2.0 & -2.23 & 1.45 & 7.5 & 0.64 & 4.08 & 15.46 & 0.163 & 29.8 & 4.0 & 25.80 & \\
\hline $\mathrm{A} 2 \ldots \ldots$ & 2.0 & -4.81 & 2.07 & 7.8 & 0.66 & 17.78 & 11.74 & 0.122 & 29.6 & 378.0 & 24.13 & \\
\hline A3 ....... & 2.0 & -3.11 & 1.66 & 7.7 & 0.64 & 5.99 & 14.66 & 0.140 & 29.9 & 6.7 & 24.81 & \\
\hline $\mathrm{A} 4$....... & 2.0 & -2.08 & 1.45 & 7.2 & 0.69 & 4.09 & 15.80 & 0.170 & 29.5 & 4.2 & 37.41 & Mask changed \\
\hline A5 ....... & 2.0 & -1.86 & 1.39 & 7.1 & 0.69 & 3.79 & 15.92 & 0.182 & 29.7 & 4.0 & 36.94 & Mask changed \\
\hline B1 ...... & 1.9 & -1.44 & 1.45 & 7.7 & 0.65 & 3.27 & 14.71 & 0.130 & 10.7 & 3.7 & 24.03 & \\
\hline B2 ........ & 1.8 & -2.21 & 1.45 & 7.4 & 0.64 & 4.17 & 14.18 & 0.159 & 29.8 & 3.8 & 25.69 & \\
\hline B3 ....... & 1.9 & -2.27 & 1.46 & 7.5 & 0.64 & 4.14 & 14.91 & 0.159 & 30.0 & 3.9 & 25.55 & \\
\hline B4 ....... & 1.9 & -1.73 & 1.35 & 7.1 & 0.68 & 3.54 & 15.44 & 0.189 & 29.6 & 3.7 & 37.32 & Mask changed \\
\hline B5 ....... & 1.0 & -1.91 & 2.15 & 9.0 & 0.65 & 10.74 & 3.38 & 0.077 & 3.6 & 3.1 & 24.01 & \\
\hline B6 ....... & 1.0 & -2.28 & 4.85 & -0.3 & 0.70 & 26.58 & 0.45 & 0.043 & 30.3 & 3.7 & 71.82 & \\
\hline $\mathrm{C} 1 \ldots \ldots$ & 2.0 & -1.35 & 1.2 & 7.0 & 0.64 & 2.64 & 16.53 & 0.233 & 29.7 & 3.2 & 28.51 & \\
\hline $\mathrm{C} 2 \ldots \ldots$ & 2.0 & -1.49 & 1.25 & 7.2 & 0.64 & 2.88 & 16.27 & 0.209 & 29.8 & 3.3 & 27.77 & \\
\hline $\mathrm{C} 3 \ldots \ldots$ & 2.0 & -1.65 & 1.3 & 7.3 & 0.64 & 3.2 & 15.95 & 0.193 & 29.8 & 3.5 & 27.14 & \\
\hline $\mathrm{C} 4 \ldots \ldots$ & 2.0 & -1.84 & 1.35 & 7.3 & 0.64 & 3.41 & 15.86 & 0.180 & 29.8 & 3.6 & 26.64 & \\
\hline $\mathrm{C} 5 \ldots \ldots$ & 2.0 & -2.03 & 1.4 & 7.4 & 0.64 & 3.75 & 15.61 & 0.171 & 29.8 & 3.8 & 26.20 & \\
\hline $\mathrm{C} 6 \ldots \ldots$ & 2.0 & -2.44 & 1.5 & 7.5 & 0.64 & 4.51 & 15.20 & 0.156 & 29.8 & 4.3 & 25.51 & \\
\hline $\mathrm{C} 7 \ldots \ldots$ & 2.0 & -2.64 & 1.55 & 7.6 & 0.64 & 5.05 & 14.91 & 0.151 & 29.8 & 4.9 & 25.22 & \\
\hline C8 ....... & 2.0 & -2.85 & 1.6 & 7.7 & 0.64 & 5.52 & 14.72 & 0.146 & 29.8 & 5.5 & 24.99 & \\
\hline C9 ....... & 2.0 & -3.06 & 1.65 & 7.7 & 0.64 & 6.19 & 14.44 & 0.142 & 29.8 & 6.6 & 24.78 & \\
\hline $\mathrm{C} 10 \ldots \ldots$ & 2.0 & -3.27 & 1.7 & 7.7 & 0.65 & 6.91 & 14.16 & 0.139 & 29.8 & 8.2 & 24.59 & \\
\hline $\mathrm{C} 11 \ldots \ldots$ & 2.0 & -3.64 & 1.8 & 7.7 & 0.65 & 8.72 & 13.59 & 0.133 & 29.6 & 14.3 & 24.27 & \\
\hline $\mathrm{C} 12 \ldots \ldots$ & 2.0 & -4.10 & 1.9 & 7.8 & 0.65 & 10.82 & 13.09 & 0.128 & 29.7 & 39.1 & 24.19 & \\
\hline $\mathrm{C} 13 \ldots \ldots$ & 2.0 & -4.51 & 2.0 & 7.8 & 0.65 & 13.84 & 12.46 & 0.124 & 29.7 & 129.7 & 24.10 & \\
\hline $\mathrm{C} 14 \ldots \ldots$ & 2.0 & -4.90 & 2.1 & 7.8 & 0.66 & 17.72 & 11.83 & 0.120 & 29.7 & 492.0 & 24.12 & \\
\hline $\mathrm{C} 15 \ldots \ldots$ & 2.0 & -5.30 & 2.2 & 7.8 & 0.66 & 22.91 & 11.21 & 0.117 & 29.7 & 2210.1 & 24.19 & \\
\hline $\mathrm{D} 1 \ldots \ldots$ & 2.0 & -3.10 & 1.45 & 10.2 & 0.55 & 5.58 & 15.02 & 0.204 & 30.6 & 7.7 & 31.10 & \\
\hline $\mathrm{D} 2 \ldots \ldots$ & 2.0 & -2.80 & 1.45 & 9.4 & 0.58 & 4.76 & 15.44 & 0.187 & 30.6 & 5.2 & 28.33 & \\
\hline D3 ....... & 2.0 & -2.50 & 1.45 & 8.4 & 0.62 & 4.5 & 15.23 & 0.175 & 30.2 & 4.4 & 26.43 & \\
\hline $\mathrm{D} 4 \ldots \ldots \ldots$ & 2.0 & -1.70 & 1.45 & 5.5 & 0.71 & 3.72 & 15.24 & 0.148 & 28.4 & 4.0 & 28.93 & \\
\hline D5 ....... & 2.0 & -3.00 & 1.5 & 9.3 & 0.59 & 5.4 & 15.15 & 0.178 & 30.4 & 6.5 & 27.56 & \\
\hline $\mathrm{E} 1 \ldots \ldots$ & 2.0 & -2.23 & 1.45 & 7.2 & 0.67 & 11.27 & 10.61 & 0.194 & 29.7 & 7.6 & 39.86 & \\
\hline E2 ....... & 2.0 & -2.05 & 1.45 & 7.8 & 0.64 & 1.95 & 23.59 & 0.125 & 29.7 & 3.0 & 45.53 & \\
\hline$F 1 \ldots \ldots$ & 2.0 & -2.07 & 1.45 & 7.5 & 0.65 & 3.93 & 15.34 & 0.146 & 23.8 & 3.9 & 25.55 & \\
\hline$F 2 \ldots \ldots$ & 2.0 & -2.34 & 1.45 & 7.5 & 0.64 & 4.19 & 15.53 & 0.168 & 35.8 & 4.1 & 25.99 & \\
\hline F3 ....... & 2.0 & -1.32 & 1.45 & 7.5 & 0.67 & 3.05 & 15.37 & 0.128 & 10.1 & 3.8 & 24.11 & \\
\hline $\mathrm{G} 1 \ldots \ldots$ & 2.0 & -3.01 & 1.64 & 7.7 & 0.64 & 5.71 & 14.76 & 0.141 & 29.9 & 5.9 & 24.91 & $R_{\min }=2 \mathrm{AU}$ \\
\hline $\mathrm{G} 2 \ldots \ldots$ & 2.0 & -2.11 & 1.45 & 8.3 & 0.60 & 3.68 & 18.82 & 0.157 & 29.8 & 3.5 & 30.73 & $R_{\max }=200 \mathrm{AU}$ \\
\hline G3 $\ldots \ldots$ & 2.0 & -2.38 & 1.45 & 7.1 & 0.67 & 4.44 & 13.41 & 0.168 & 29.9 & 4.5 & 22.75 & $R_{\max }^{\max }=280 \mathrm{AU}$ \\
\hline $\mathrm{G} 4 \ldots \ldots$ & 2.0 & -2.47 & 1.45 & 6.3 & 0.71 & 5.33 & 10.88 & 0.171 & 30.1 & 5.2 & 21.66 & $R_{\max }=340 \mathrm{AU}$ \\
\hline G5 ....... & 2.0 & -2.60 & 1.45 & 5.5 & 0.74 & 7.15 & 8.40 & 0.185 & 30.0 & 6.4 & 21.42 & $R_{\max }=425 \mathrm{AU}$ \\
\hline G6.......... & 2.0 & -2.55 & 1.45 & 4.3 & 0.78 & 9.49 & 6.21 & 0.187 & 29.9 & 7.8 & 22.02 & $R_{\max }^{\max }=525 \mathrm{AU}$ \\
\hline $\mathrm{H} 1 \ldots . .$. & 2.0 & -2.55 & 2.18 & 3.0 & 0.76 & 7.44 & 10.37 & 0.084 & 29.9 & 5.3 & 42.75 & \\
\hline $\mathrm{H} 2 \ldots \ldots$ & 2.0 & -2.17 & 1.65 & 5.0 & 0.71 & 4.57 & 14.20 & 0.121 & 29.9 & 4.3 & 30.12 & \\
\hline $\mathrm{H} 3 \ldots \ldots$ & 2.0 & -3.92 & 1.74 & 9.0 & 0.61 & 8.23 & 14.51 & 0.147 & 29.9 & 31.4 & 25.21 & \\
\hline $\mathrm{H} 4 \ldots \ldots$ & 2.0 & -6.79 & 1.99 & 13.0 & 0.51 & 17.02 & 16.09 & 0.163 & 25.5 & $8 \times 10^{6}$ & 34.01 & \\
\hline $\mathrm{I} 1 \ldots \ldots \ldots$ & 2.0 & -5.83 & 1.82 & 13.2 & 0.30 & 6.25 & 18.73 & 0.173 & 30.1 & $7 \times 10^{4}$ & 44.04 & \\
\hline $\mathrm{I} 2 \ldots \ldots \ldots$ & 2.0 & -3.93 & 1.71 & 9.4 & 0.55 & 6.35 & 15.60 & 0.148 & 30.0 & 28.6 & 27.75 & \\
\hline $\mathrm{I} 3 \ldots \ldots \ldots$ & 2.0 & -2.54 & 1.71 & 5.9 & 0.75 & 6.15 & 13.63 & 0.130 & 29.7 & 5.3 & 28.88 & \\
\hline $\mathrm{I} 4 \ldots \ldots \ldots$ & 2.0 & -1.09 & 1.2 & 4.1 & 0.90 & 2.58 & 18.14 & 0.448 & 30.1 & 3.7 & 47.30 & \\
\hline I5 ........ & 2.0 & -3.00 & 1.4 & 11.6 & 0.30 & 2.55 & 18.88 & 0.204 & 30.0 & 4.2 & 47.47 & \\
\hline $\mathrm{J} 1 \ldots \ldots \ldots$ & 2.0 & -2.23 & 1.45 & 7.4 & 0.86 & 6.18 & 15.54 & 0.038 & 47.0 & 6.1 & 15.39 & F555W \\
\hline $\mathrm{J} 2 \ldots \ldots \ldots$ & 2.0 & -2.23 & 1.45 & 7.4 & 0.86 & 6.22 & 15.54 & 0.040 & 47.3 & 6.1 & 15.39 & F555W \\
\hline $\mathrm{J} 3 \ldots \ldots \ldots$ & 2.0 & -2.23 & 1.45 & 7.4 & 0.69 & 4.34 & 15.54 & 0.086 & 33.0 & 4.3 & 12.10 & F675W \\
\hline $\mathrm{J} 4 \ldots \ldots \ldots$ & 2.0 & -2.23 & 1.45 & 7.4 & 0.80 & 4.8 & 15.54 & 0.090 & 36.6 & 4.7 & 12.31 & F675W \\
\hline $\mathrm{J} 5 \ldots \ldots$ & 2.0 & -2.23 & 1.45 & 7.4 & 0.64 & 4.03 & 15.54 & 0.162 & 30.7 & 4.0 & 25.46 & F814W \\
\hline $\mathrm{K} 1 \ldots \ldots$ & 2.0 & -2.76 & 1.45 & 9.2 & 0.59 & 5.24 & 14.86 & 0.200 & 30.6 & 5.4 & 28.89 & \\
\hline $\mathrm{K} 2 \ldots \ldots$ & 2.0 & -1.63 & 1.45 & 4.6 & 0.66 & 2.48 & 17.34 & 0.100 & 28.4 & 3.1 & 42.00 & \\
\hline $\mathrm{K} 3 \ldots \ldots$ & 2.0 & -1.44 & 1.45 & -0.4 & 0.60 & 0.68 & 27.67 & 0.010 & 28.9 & 1.4 & 202.3 & \\
\hline $\mathrm{L} 1 \ldots \ldots$. & 2.0 & -1.95 & 1.45 & 6.1 & 0.62 & 1.8 & 20.59 & 0.116 & 29.3 & 2.4 & 38.05 & \\
\hline $\mathrm{L} 2 \ldots \ldots$ & 2.0 & -2.48 & 1.45 & 8.3 & 0.83 & 5.93 & 14.11 & 0.183 & 30.2 & 5.4 & 27.35 & \\
\hline L3 ........ & 2.0 & -3.83 & 1.45 & 7.6 & 0.61 & 9.01 & 14.90 & 0.280 & $>92$ & 64.6 & 28.64 & \\
\hline
\end{tabular}




$$
\sigma_{\mathrm{tot}}^{2}=\left[2 \rho_{\mathrm{read}}^{2}+\left(\sigma_{\mathrm{flat}} G I\right)^{2}+G I+D T\right] / G^{2} .
$$

For good physical reasons, some parts of the image are not explicable within the context of the simple axisymmetric model. As discussed next, their contribution to the merit function is suppressed in order to avoid skewing the results.

The models tend to fit the high signal level regions well, but do a poorer job where the signal level is lower, but still significant. In a case in which a model does not completely fit data to within its signal-to-noise limitations, the fit parameters are uncertain because they depend on the weighting scheme used. We have chosen to treat each pixel with its correct statistical weight, but to give some regions of the image zero weight because we have reason to believe that the model is inadequate there, and we wish to avoid skewing the results. The excluded regions can be seen in the top right panel of Figure 6. They are a region that includes emission from the jet, and a region in the north-northeast reflection nebula that is anomalously bright. This bright region is not axisymmetric and cannot be explained by our models. It may be due to material falling onto the disk from the envelope (although the low reddening argues against the existence of a signficant extended envelope). Alternatively, it may be due to an instability in the disk. Specifically, warmer regions of the disk will expand and then catch more heat from the central source, tending to make them even warmer. If only the jet region is excluded from the fit, the derived parameters do not change by large amounts, but the quality of the fit as measured by $\chi^{2}$ decreases markedly, as shown in Table 3 by models A4, A5, and B4, which have their $\chi^{2}$ values in italics to indicate that a different mask was used. These models can be compared directly to models A1, A3, and B3, respectively. Even outside the excluded region, the models do not provide a statistically perfect representation of the data within noise limitations, although the proportional errors are generally small. Part of the reason for the remaining discrepancy is undoubtedly the singlescattering approximation, as demonstrated above.

\subsection{Disk Vertical Profile}

The form of the disk's vertical profile is well constrained by the data to be close to $\xi=2$ (a Gaussian), and $\xi=1$ (an exponential) is excluded. Model B1 in Table 3 shows the result of a fit with only $\beta$ constrained by theoretical prejudice to 1.45 . It can be seen that the vertical profile is very close to a Gaussian. This conclusion is insensitive to the presence of extinction constraints (B2); the presence of a constraint to the canonical radial power law (B3); and the masking choice (B4). On the other hand, if the disk profile is forced to be exponential, a satisfactory fit is possible, but only with very small extinction so that the central star is clearly visible in the image (B5). When the extinction is constrained, the best result is a very poor fit (B6).

There are strong theoretical reasons to expect a Gaussian profile, but we believe that this is the first time the profile has been measured directly. A Gaussian vertical distribution of density is valid for a hydrostatic vertically isothermal non-self-gravitating gaseous disk (Weidenschilling \& Cuzzi 1993). The accretion disk is expected to contain gas, and the scattering particles are probably well coupled to the gas. As discussed in the section below on the disk mass, the midplane of the disk may be cooler, but we expect the entire observable disk to be vertically isothermal because the near-infrared photons that predominantly heat the disk penetrate further than the observed scattered light. A Gaussian profile also holds if the particles have decoupled from the gas (or no gas is present), provided that they are of roughly equal masses and in energy equipartition. We do not expect that this is the case for $\mathrm{HH} 30$, but we cannot rule it out on the basis of the observed profile. A pressuresupported disk that is dominated by the disk self-gravity would have an exponential vertical profile, and this is ruled out. The agreement between the theoretical expectation and observed best-fit profile is so good that we have constrained the profile to be exactly Gaussian in all the other fits (and in particular in the canonical fit presented above).

\subsection{Disk Geometry}

The disk geometry is characterized by the radial powerlaw index of the disk thickness, $\beta$, and the radial power-law index for the central density $\alpha$. As observed above, a range of solutions is possible with different values of these parameters. Nevertheless, for any fixed extinction to the central object the solution is constrained to lie close to an approximately straight line in the $\alpha-\beta$ plane. Distinctly worse fits occur if $\beta$ is constrained to be less than 1.2 or greater than 2.2. So $\beta$ can be assumed confidently to lie in this range. For a variety of values of $\beta$, and with $A_{I}=30 \mathrm{mag}$, fits were run to derive the corresponding best-fit $\alpha$. The results are presented as runs $\mathrm{C} 1-\mathrm{C} 15$ in Table 3 and plotted in Figure 8.

In runs D1-D4 of Table $3, \beta$ is constrained to be equal to a particular value (1.45), and $\alpha$ is varied away from the best-fit value ( -2.2 for run $A 1)$. Runs such as these allowed the construction of the shaded region in Figure 8 that represents the approximate range of acceptable values $\left(\chi^{2}\right.$ less than 3.5 bigger than the minimum). Points near the boundary of the shaded region are distinctly worse fits. A number of theoretical limits and loci are also shown in the figure, and these are discussed next.

If the disk is pressure supported and vertically isothermal with temperature $T(r)$, the resulting Gaussian vertical density distribution has scale height $H(r)=\left[k T(r) r^{3} / G M m\right]^{1 / 2}$,

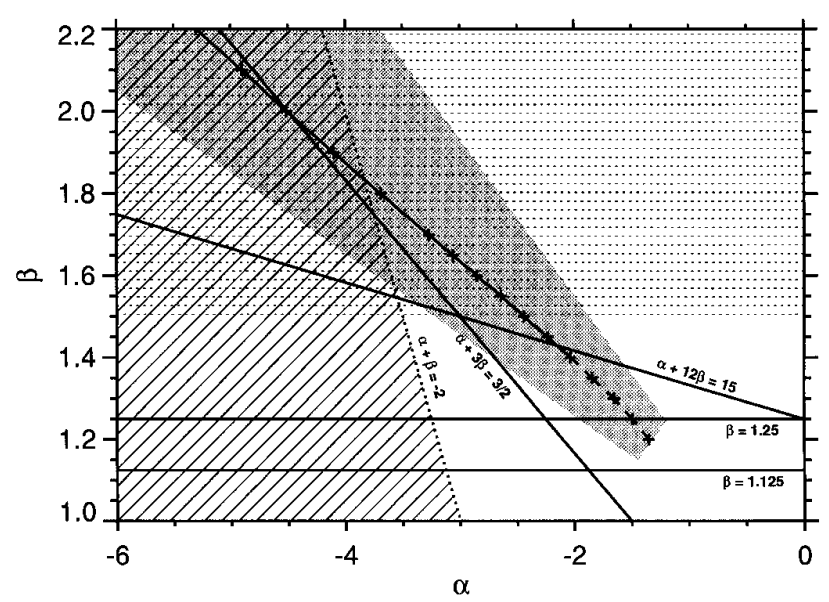

FIG. 8. $-\alpha-\beta$ plane. The shaded region is consistent with the data with $30 \mathrm{mag}$ of extinction. The best-fit points are plotted in the center of this region. With $60 \mathrm{mag}$ of extinction, the whole region can be moved to the left by about 0.6 in $\alpha$. The hatched region corresponds to large disk masses. The dotted region corresponds to a disk that is growing in temperature with radius or is not pressure supported. Lines correspond to various theoretical cases (see text). 
with $M$ and $m$ being the protostellar and mean molecular masses, respectively. Thus, $T(r)=T\left(r_{0}\right)\left(r / r_{0}\right)^{2 \beta-3}$. Different assumptions about the disk heating mechanisms imply corresponding temperature radial power laws and hence different values for $\beta$.

A radially isothermal disk would have $\beta$ of 1.5 . If $\beta$ is larger than 1.5, and the disk is in local pressure equilibrium, then the temperature would be increasing with radius. For such values of $\beta$, it is more reasonable to conclude that the material that is seen is not in pressure equilibrium and instead is infalling envelope material. Therefore, the top half of the diagram has been shaded. We cannot exclude this region on the basis of observations, although we do have indirect evidence for pressure support. As discussed below, there is a self-consistent solution at $\beta=1.45$ that is right for a disk that decreases slowly in temperature. When $\beta>2$, the derived disk mass becomes very large, and this is inconsistent with the millimeter mass measurements.

A centrally heated optically thin disk will have a temperature that falls as $r^{-1 / 2}$ so $\beta=1.25$. Although the fits do not exclude this value of $\beta$, this particular model can be excluded because the disk is clearly not optically thin.

A geometrically thin but optically thick plane disk heated by a thicker central source has a temperature that falls as $r^{-3 / 4}$, and so $\beta=1.125$. An identical power law applies if the disk is a steady state viscous accretion disk with the local temperature set by the local viscous dissipation of orbital energy (see, for example, Lin \& Papaloizou 1985). It also applies to a good approximation in full time-dependent evolutionary calculations (Ruden \& Pollack 1991). The $\beta=1.125$ locus is plotted near the bottom of the figure, and as it does not intersect the shaded region, it can be excluded confidently by the HST data.

If the disk is geometrically thin (so $h \ll r$ ), optically thick to absorption of visible and near-infrared light, but optically thin to emission of far-infrared light, the equation for local thermodynamic equilibrium is

$$
4 \Sigma(r) \kappa(T) \sigma T^{4}=a\left(L_{*} / 4 \pi r^{2}\right)(\beta-1) H(r) / r,
$$

where $4 \kappa(T)$ is the Planck-averaged emissivity per unit mass, which scales as $T^{2}$ for small grains at temperatures below $50 \mathrm{~K}$ (Draine $\&$ Lee 1984). The factor $(\beta-1) H(r) / r$ is the inclination of the central illumination to the local disk tangent in the small angle approximation. Equating the exponents of $r$ on the two sides of this equation, and assuming pressure support (so $T$ is proportional to $r^{2 \beta-3}$ ) gives $\alpha+\beta+6(2 \beta-3)=\beta-3$, or $\alpha+12 \beta=15$. This important case is plotted in Figure 8 . It intercepts the set of possible solutions at $\beta=1.45$, which is why that particular solution was chosen as the reference case.

A flared, locally isothermal and optically thick disk that is optically thick to emission will have $4 \Sigma(r) \kappa(T)$ in the above equation replaced with a constant emissivity and therefore a temperature that falls as $r^{(\beta-3) / 4}$. If it is also pressure supported, we find that $\beta=1.28$. This power law is consistent with the locus of possible fits with $\alpha$ in the range -2 to -1 , but the disk model fit appropriate to this flaring function $(\mathrm{C} 9)$ is thin to emission $\left(\tau \sim 10^{-2}\right)$, so this case can be excluded.

Another important case is an accretion disk with a kinematic viscosity proportional to the scale height times the sound speed (an alpha disk). In this case, the mass accretion rate can be computed and is only a constant throughout the disk if $\alpha+3 \beta=3 / 2$. This line is also plotted in Figure 7 .
Points to the right of this line (such as the reference case) represent cases in which the accretion rate is an increasing function of radius. There is no particular reason why the accretion rate should be a constant function of radius even for a steady state disk, because infalling envelope material can cause the accretion rate to be a decreasing function of radius, while mass loss from the disk surface (due to jet entrainment or a thermally induced wind) can cause the opposite. However, a particularly simple and important case is when the disk is a pressure-supported alpha disk that is thin to emission and has a constant accretion rate. This case occurs at the intersection of the lines $\alpha+12 \beta=15$ and $\alpha+3 \beta=3 / 2$, when $\alpha=-3$ and $\beta=1.5$, so the disk must also be isothermal. The result of a fit to this case is given in run D5 of Table 3.

Infrared spectra of $\mathrm{T}$ Tauri stars typically indicate that the outer disk is warmer than expected from radiative equilibrium with the central source for a flat disk, perhaps due to flaring or heating by scattered light from the infalling envelope. Beckwith et al. (1990) find radial temperature power-law indices in the range -0.47 to -0.75 by fitting infrared spectral energy distributions, with a peak around -0.57 , corresponding to $\beta$ peaking at 1.21 and in the range $1.12-1.26$. If the same result holds for HH 30 , the fits show that $\beta$ must lie near the upper limit derived by this method.

\subsection{Scale Height}

As can be seen from Table 3 , although a wide range of models can fit the data reasonably well, all the models have very similar scale heights at $100 \mathrm{AU}$, of about $15.5 \mathrm{AU}$. If the scale height is constrained to values $30 \%$ smaller, or $50 \%$ larger as in runs E1 and E2 of Table 3, the fit is much worse at fixed $\beta=1.45$. The apparent variation of scale height with $\beta$ in runs $\mathrm{C} 1-\mathrm{C} 15$ of Table 3 is caused by the particular reference radius of $100 \mathrm{AU}$ that was chosen. If instead a reference radius of $138 \mathrm{AU}$ is used, the scale height there is $24.5 \pm 0.03 \mathrm{AU}$ for all values of $\beta$.

A central mass $M$, and a mean molecular mass of 2 AMU, imply a temperature at $100 \mathrm{AU}$ of $T\left(r_{0}\right)=50.5$ $\left(M / M_{\odot}\right)\left[H\left(r_{0}\right) / 15.5\right]^{2}$. Then the equation for radiative equilibrium can be solved to derive the luminosity of the central source:

$$
\begin{aligned}
L_{*} & =16 \pi r_{0}^{3} \Sigma\left(r_{0}\right) \kappa(T) \sigma T^{4} / A(\beta-1) H\left(r_{0}\right) \\
& =1.0 L_{\odot}\left[H\left(r_{0}\right) / 15.5\right]^{12}\left(M / 0.67 M_{\odot}\right)^{6} .
\end{aligned}
$$

The result is also proportional to the measured central density and the Planck-averaged opacity, which has been taken as $\kappa(T)=5.68 \times 10^{-4} T^{2} \mathrm{~cm}^{2} \mathrm{~g}^{-1}$. Draine \& Lee give $\kappa(T)=6 \times 10^{-4} T^{2} \mathrm{~cm}^{2} \mathrm{~g}^{-1}$; the adopted value corresponds to an opacity of $0.1(250 \mu \mathrm{m} / \lambda)^{2}$. The luminosity is $10 L_{\odot}$ for a solar mass central object, which is uncomfortably large, but a statistically more likely central mass of $0.67 M_{\odot}$ implies $1 L_{\odot}$. With this mass, the temperature at $100 \mathrm{AU}$ is $34 \mathrm{~K}$, and the Planck-averaged normal optical depth at 100 AU is 0.016 , which justifies the assumption that the disk is thin to emission. The disk becomes optically thick to emission in regions in which it is warmer and denser, with an optical depth of unity at $2.7 \mathrm{AU}$. It can be expected to transition to a $\beta=1.28$ disk near this point. If the mass accretion rate exceeds $2.3 \times 10^{-8} \mathrm{AU} \mathrm{yr}^{-1}$, then the accretion luminosity will exceed the central heating at this radius, and the disk will transition to a $\beta=1.125$ disk without 
passing through the optically thick case. For all reasonable values of the accretion rate, optical parameters, and central mass and luminosity, the disk is thin to emission and not predominantly heated by local accretion luminosity to below our resolution limit.

The self-shielding of the disk is essential in order to obtain a reasonable central source luminosity. An unshielded grain at $100 \mathrm{AU}$ would be at $71 \mathrm{~K}$, and the central luminosity would have to be $0.012 L_{\odot}$ in order to lower the temperature to the observed value. Similarly, the fact that the disk is optically thin to emission is also necessary. An efficient radiator would reach only $14 \mathrm{~K}$ at 100 $\mathrm{AU}$, and the central luminosity would have to be 32.1 times solar to heat it sufficiently.

\subsection{Extinction}

The extinction toward the star itself is not constrained directly by the observations, as the stellar type and luminosity are not known. Extinction along the line of sight to the star can be estimated from the optical and infrared colors of $\mathrm{HH} 30$. One way to estimate the stellar extinction is to compare near-infrared photometry of HH 30 to other $\mathrm{T}$ Tauri stars and Herbig-Haro exciting stars. In TaurusAuriga, a typical T Tauri star has an observed $K$ magnitude of about 9 (Kenyon \& Hartmann 1995; Strom et al. 1989), and the theoretical stellar "birthline" corresponds to $K$ magnitudes in the range 7.5-9.7 (Stahler 1983). HH 30's $K$ magnitude is 13.2 (Vrba et al. 1985), or at least 4 mag fainter than typical for other objects of its class. If this difference was due to reddening, then the corresponding extinction by standard interstellar grains at $0.8 \mu \mathrm{m}(\mathrm{F} 814 \mathrm{~W})$ would be at least 24 mag. This estimate may be considered a lower limit, as accretion luminosity is likely to substantially augment the intrinsic stellar luminosity at $2 \mu \mathrm{m}$, and because scattered light at $K$ may be making a large contribution to the observed flux, or even explain all of it. In this case, the extinction may be many tens of magnitudes larger than the lower limit estimated here.

However, the extinction derived from the models is based on an extrapolation to denser regions of the disk (on the line of sight to the central object) of the densities inferred for the observed scattering regions. The inner regions of the disk are shielded heavily from the central object and may therefore be colder and denser than this extrapolation would indicate. Thereore, although the observational constraint is that there should be at least $24 \mathrm{mag}$ of extinction, it is possible that extrapolating the correct model of the scattering region would predict less.

For our models, we have usually constrained the line-ofsight extinction to be $A_{I}=30 \mathrm{mag}$. This constraint is consistent with the lower limit of $A_{I}=24 \mathrm{mag}$ derived above and equal to the measured extinction for HL Tau (Beckwith \& Birk 1995; Stapelfeldt et al. 1995; Roddier 1996). Varying the extinction over the small range from 24 to 36 mag runs F1-F2) does not seem to change significantly the derived parameters. For example, the disk mass varies by only $\pm 3 \%$ from the nominal value. As the extinction is lowered a slight increase in $\alpha$ (putting more material near the star) can compensate without changing the other parameters very much.

On the other hand, if the extinction is unconstrained, the fit tends to have only about 10 mag extinction (run F3). This case is directly ruled out unless a denser core region of the disk exists because the central source then becomes just visible in the image, and the $K$-band flux would be much higher. Even in this case, most of the other fit parameters are changed by only at most a few tens of percent.

If the (extrapolated) extinction is as high as $60 \mathrm{mag}$, the best fits still lie within the left part of the shaded region in Figure 8. If, in addition, the solution is constrained to lie on the line $\alpha+12 \beta=15$ to be consistent with thermal equilibrium, then the disk is isothermal and has a constant accretion rate (like run D5). If the (extrapolated) extinction significantly exceeds $60 \mathrm{mag}$, it is hard to understand the temperature profile in the disk, which would probably have to increase with radius.

In conclusion, the central radiation source is not seen in the images, and so the extinction to this source is not well constrained. However, we believe it is at least $24 \mathrm{mag}$. The extinction extrapolated from the models ranges from 10 to about $60 \mathrm{mag}$ and probably underestimates the true extinction. Within this range of models, the values for other parameters are largely insensitive to the amount of extinction.

\subsection{Disk Size Limits}

Most of the models are insensitive to the choice of $r_{\min }$, the inner disk cutoff. On the other hand, $r_{\max }$ is constrained by the observations to be greater than about $250 \mathrm{AU}$.

If $r_{\text {min }}$ is quadrupled from $0.5 \mathrm{AU}$ to $2 \mathrm{AU}$, the result is shown in run G1 of Table 3. This result is almost identical to the result in run A3 (except for an increase of 0.1 in $\alpha$ to compensate for the reduced optical depth to the source contributed by the excluded inner region of the disk). The reason is that light that strikes the outer disk generally passes through this inner region at many scale heights because of the disk flaring, and even though the central density is large, the optical depth at higher latitudes is very low.

The inner cutoff should be set by the disk temperature profile, which is poorly determined. Significant ices will sublimate at temperatures of about $80 \mathrm{~K}$, while on the other hand silicate grains will survive to perhaps $1200 \mathrm{~K}$. The density and temperature profile deduced from the outer disk implies a temperature of $50 \mathrm{~K}$ at $2.7 \mathrm{AU}$, where the disk becomes optically thick to its emitted radiation (for a 0.67 $M_{\odot}$ central object). The disk temperature then grows to 80 $\mathrm{K}$ by $1.2 \mathrm{AU}$. However, accretion energy will have a comparable heating effect in the region near the cutoff for accretion rates greater than $10^{-8} M_{\odot} \mathrm{yr}^{-1}$.

The accretion rate can be constrained by two separate arguments. First, it is unlikely that the total luminosity of the $\mathrm{HH} 30$ system could exceed several $L_{\odot}$ without producing large infrared excesses that are not observed. This provides an upper bound to the total accretion luminosity $L=$ $G M \dot{M} / R$ and mass accretion rate. For accretion to a 0.5 $M_{\odot}$ star of radius $3 L_{\odot}, \dot{M}$ is limited to less than about $10^{-6} M_{\odot} \mathrm{yr}^{-1}$. Second, the mass outflow rate in the bipolar jets has been estimated at about $5 \times 10^{-10} M_{\odot} \mathrm{yr}^{-1}$ from emission-line diagnostics (Mundt et al. 1990). Revising this value to reflect the ionization fraction of a few percent now thought to be prevalent in Herbig-Haro jets, we obtain $5 \times 10^{-9} M_{\odot} \mathrm{yr}^{-1}$. If $10 \%$ of the accreting material is diverted into the outflow, the net mass accretion rate is about $5 \times 10^{-8} M_{\odot} \mathrm{yr}^{-1}$.

The maximum accretion rate of $10^{-6} M_{\odot} \mathrm{yr}^{-1}$ raises the disk temperature to $80 \mathrm{~K}$ at $4.9 \mathrm{AU}$ becuse the disk is an efficient radiator at this temperature (optically thick), and to 
$1200 \mathrm{~K}$ at $0.13 \mathrm{AU}$. Beckwith et al. (1990) finds a temperature between 100 and $300 \mathrm{~K}$ at 1 AU from other $\mathrm{T}$ Tauri stars by fitting the infrared spectral energy distribution (with higher values when the accretion rate is high), and this seems consistent with our results given the low accretion rate of $\mathrm{HH} 30$.

We conclude that $r_{\text {min }}$ is probably betwen $1.2 \mathrm{AU}$ and 4.9 $\mathrm{AU}$ for grains that are destroyed at $80 \mathrm{~K}$ and perhaps 0.13 AU for silicate grains, but the results are insensitive to the value chosen.

$R_{\max }$ is constrained to be greater than $250 \mathrm{AU}$ by the visible extent of the nebulae. If it is cut to $200 \mathrm{AU}$, the fit is much worse $(\mathrm{G} 2)$. If it is increased, then the $\chi^{2}$ merit function continues to decrease to a minimum at about $400 \mathrm{AU}$ but at a much slower rate (G2-G6 have $r_{\min }=200,280,340$, 425 , and $525 \mathrm{AU}$, respectively). On the other hand, due to extinction in the extra outer regions, the brightness of the counternebula decreases, so the ratio of the nebular brightnesses comes out increasingly wrong. The best-fit model has a total normal optical depth of only 1.3 at $250 \mathrm{AU}$, and much less in the region that is not shielded by the inner disk. Thus, the outer nebula is very difficult to observe directly because it is becoming optically thin. We have adopted an outer cutoff $r_{\max }$ of 250 AU because this covers the visible extent of the nebula, is consistent with size estimates for other protostellar disks, and produces the best fit to the counter nebula.

\subsection{Disk Axis}

The disk axis is constrained by the fits to the images to lie at position angle $32.2 \pm 1.0$. This is consistent with both the jet axis near the star of 31.3 and the average jet axis of 30.8. Thus, the jet is within a degree of perpendicular to the disk in the plane of the sky.

The models also constrain the inclination of the jet to the plane of the sky to be $8^{\circ} \pm 2^{\circ}$. Runs $\mathrm{H} 1-\mathrm{H} 4$ of Table 3 and the third row of Figure 7 illustrate the problems that occur if the inclination is outside this range. The primary problem is that either the shape or the brightness of the counternebula is incorrect. However, there are two important caveats that apply to this result. First, the observed nebula/ counternebula brightness ratio is not temporally constant, which shows that the central source is not isotropic, and some of the brightness difference between the nebulae may be accounted for. Second and more importantly, we will develop arguments that the disk is not vertically isothermal. The effect of this is that much more cold material is in the line of sight to the counternebula than the simple model presented here would suggest. This, in turn, means that the disk inclination will be less. We conclude that our measured inclination should be treated as an upper bound. There is evidence in the images for this colder and denser region. The counternebula has finger-like extensions to its corners farthest from the star. These regions are not covered so much by the denser central regions, and that is why they appear brighter. Such fingers cannot be produced by our simple isothermal disk model.

The jet axis is also constrained kinematically by combining our proper-motion measurements with the radial velocity measurement of Cohen \& Jones (1987). The result is that the jet axis is inclined $0^{\circ} \pm 3^{\circ}$ into the plane of the sky. Mundt et al. (1990) present higher signal-to-noise ratio spectra and place the jet axis within 0.5 of the plane of the sky.
In conclusion, the jet is perpendicular to the disk to within a few degrees in three dimensions.

\subsection{Grain-Scattering Properties}

The optical scattering asymmetry properties of the grains in the nebula are constrained significantly by the data. The asymmetry parameter $g=\langle\cos (\theta)\rangle$ is in the range $0.64 \pm 0.06$ for all the good fits in Table 3 . The only significant exception is if the outer disk cutoff is larger than 340 $\mathrm{AU}$, when it can be as large as 0.78 . We have attempted to force $g$ to values outside this range, and the results are invariably poor. Runs I1-I4 show the result, and examples are reproduced in Figure 7. This value is much larger than the prediction in White (1979) of 0.3 based on the theoretical MRN mixture (Mathis et al. 1977). The unconstrained fit to 0.3 in run $\mathrm{I} 1$ produces a large disk mass, and a very poor fit. If $\alpha$ is constrained to -3 in order to control the mass, the fit is still unacceptable. Our larger value is in agreement with the value of 0.7 measured for interstellar dust by Witt et al. (1990). The original MRN mixture did not have any particles larger than $0.25 \mu \mathrm{m}$, and this is probably the source of the discrepancy.

The I-band image has been used to fix the disk density distribution. This does not change if the bandpass is altered, so a number of runs were performed with the density fixed in order to investigate the wavelength dependence of the grain optical properties. Runs $\mathrm{J} 1, \mathrm{~J} 3$, and $\mathrm{J} 5$ are the result of fits to the F555W, F675W, and F814W ( $V, R$, and $I)$ bandpasses. The goodness-of-fit statistic is much better in the first two cases simply because the signal-to-noise ratio of this data is lower. Here $g$ is seen to increase at bluer wavelengths, reaching 0.69 at $R$ and 0.86 at $V$. We obtain the same results if a similar set of runs is performed at 60 mag of extinction, instead of 30 , so the derived grain properties do not depend at all on the assumed extinction to the central source.

From Table 1, the nebulae differ in brightness, but in the 1994 data there is almost no color difference. The integrated brightness difference is within the range $2.70 \pm 0.05 \mathrm{mag}$, for all three bandpasses. In our models, this is explained by a balance between extra extinction on the south-southwest nebula, and more efficient wide-angle scattering for shorter wavelengths. We do find evidence for a standard interstellar extinction law in much of the nebula. However, it is also possible that the denser material responsible for dimming the south-southwest nebula consists of larger grains and hence has more neutral extinction. The opacity is expected to vary with wavelength but is fixed in the fitting software, so instead, the central density is allowed to vary. Only the product of these two parameters is a measurable. At the effective wavelengths of the $V, R$, and $I$ filters, $A_{B} / E(B-V)$ for a standard interstellar extinction law (Cardelli, Clayton, $\&$ Mathis 1989) is 3.20, 2.52, and 2.03, respectively. Therefore, an extinction of $30 \mathrm{mag}$ in the $I$ bandpass corresponds to $37.2 \mathrm{mag}$ and $47.2 \mathrm{mag}$, respectively, in the $R$ and $V$ bandpasses. If the grains follow an interstellar extinction law, then the midplane reference density $\rho_{0}$ should scale similarly between the model fits to the F814W image and the fits to the F675W and F555W images with otherwise identical geometric parameters. Between runs $\mathrm{J} 1$ and $\mathrm{J} 5, \rho_{0}$ changes by a factor of 1.53 , corresponding to an extinction for the F555W bandpass of $47 \mathrm{mag}$. This remarkable agreement shows that between the $V$ and $I$ bandpasses, the grains appear to have interstellar properties to high accu- 
racy. The derived extinction in the $R$ bandpass is 33.0. This is not such a consistent result, although this image is particularly contaminated with emission lines, and this may have skewed the result. Runs J2 and J4 were performed to fix the extinction (hence the opacity, as the result of the geometry is fixed) at the nominal values. The results for the scattering asymmetry and central source brightness are unchanged.

Finally, an indication of the color and brightness of the central source is available. Its brightness compared to the sun is 0.039 in $V, 0.088$ in $R$, and 0.162 in $I$. All these quantities should be understood to be proportional to the albedo $a / 0.5$. Thus, the results are limited by the uncertainty in the assumed albedo and its wavelength dependence, but the albedo intensity product is quite well constrained. If the extinction is constrained instead to $60 \mathrm{mag}$, the result is changed very little, and this difference is quoted as the error in Table 4. If the source brightness is forced to vary significantly as in runs $\mathrm{K} 1-\mathrm{K} 3$, the fit is much worse. As discussed above, we expect a bolometric luminosity of about solar, so the central source is very red and emits most of its energy in the infrared. These results are summarized in Table 4, together with the measured absolute source magnitudes.

\subsection{Disk Mass}

The disk mass is a derived parameter and not separately free to change. It normally depends only on the outer disk radius, but if $\gamma=\alpha+\beta+2$ is negative, it becomes sensitive to the inner cutoff instead. These cases are probably unphysical because the corresponding disk mass estimates become very large. In Figure 8 this region is hatched. In most of the models that fit the data well, $\gamma$ is positive and the resulting disk mass depends on the outer cutoff. As the disk has a Gaussian density profile, the central mass must exceed the disk mass by at least the disk aspect ratio. On this basis alone, we would therefore expect the disk mass to be less than $10 \%$ of solar, assuming the central object is not several solar masses.

The best-fit model suggests that the circumstellar disk contains $4 \times 10^{-4} M_{\odot}$ of the dust and gas. Larger masses $\left(>10^{-3} M_{\odot}\right)$ produce an absorption lane that is too wide, unless the disk is not assumed to lie on the thermal equilibrium line. Runs L1-L3 show that if the mass is constrained to be significantly different, the fit quality deteriorates rapidly. This mass determined from the optical images is about a factor of 10 smaller than that estimated using the $1.3 \mathrm{~mm}$ continuum flux. The difference is perhaps acceptable, given the large uncertainties in the $0.8 \mu \mathrm{m}$ opacities and millimeter wave emissivities for the grains. However, it is possible to reconcile these results completely by examining our assumption more carefully.

The disk is isothermal at a given radius to a good approximation throughout the region that is seen in scattered light because most of the heating is done by longer wavelength photons that will penetrate the scattering regions well. On the other hand, the mixing time in the disk is comparable to or greater than the orbital time, while the thermal time constant from grain radiation is less than a year. Thus, the disk midplane region is probably much colder and can contain significantly more mass than the amount extrapolated from visible regions. This shielded region of the disk is heated by long-wavelength photons emitted near the observed disk surface. In principle, a full self-consistent solution for the radial and vertical disk temperature should be obtained, but a crude correction can be estimated by assuming that the disk at each radius consists of three vertical isothermal regions: The bottom and top, which start at height $\pm H_{\tau}$ and are heated to $T(r)$ by the central source as before; and a shielded center, which is at a temperature $T_{1}(r)$, with corresponding scale height $H_{1}(r)$. The equation for thermal equilibrium for the top and bottom surface is the same as before (except that the mass surface density should be replaced by the illuminated mass, but the temperature only changes by the one-sixth power of this correction factor, which is of order unity, so we neglect this difference). Thermal equilibrium for the shielded region gives $\sigma T_{1}^{6}=$ $A\left(L_{*} / 4 \pi r^{3}\right)(\beta-1) H(r) T^{2}$, which can be simplified further to give $T_{1}=(4 \tau)^{1 / 6} T$. The Planck-averaged normal optical depth $\tau$ is about 0.014 at $100 \mathrm{AU}$, so the shielded temperature there is $21 \mathrm{~K}$, as opposed to $34 \mathrm{~K}$ for the observed outer regions. By integrating the equation for pressure support, and requiring pressure continuity at the temperature transition, it can be seen that in the shielded region, the density is increased by a factor $\left(T / T_{1}\right) \exp$ $\left[\left(H_{\tau}^{2}-z^{1}\right)\left(H^{2}-H_{1}^{2}\right) / 2 H^{2} H_{1}^{2}\right]$ compared to an extrapolation for the observed region. The value of this factor depends sensitively on the unknown transition height, $H_{\tau}$, at which the disk becomes optically thick to the incoming photons from the central source. $H_{\tau}$ can be estimated by direct integration of the density distribution if an average opacity $\kappa_{*}$ to the heating photons is assumed. We find that $H_{\tau}=2^{1 / 2} H \mathrm{ERFC}^{-1}\left[2^{1 / 2}(\beta-1)\left(r / r_{0}\right)^{\alpha} / \pi^{1 / 2} r \rho_{0} \kappa_{*}\right]$. To get an idea of the required correction, we assume that $\kappa_{*}=40$ $\mathrm{cm}^{2} \mathrm{~g}^{-1}$. Then $H_{\tau}$ is zero at radii less than $50 \mathrm{AU}$ (no correction is necessary). $H_{\tau}$ grows to $1.45 H$ at $100 \mathrm{AU}$, where the central density is enhanced by a factor of 2.9. At the outer cutoff of $250 \mathrm{AU}, H_{\tau}=2.73 \mathrm{H}$, and the central density is enhanced by a factor of 53. A numerical integration shows that the overall disk mass is enhanced by a factor of 13.4. If $\kappa_{*}=50 \mathrm{~cm}^{2} \mathrm{~g}^{-1}$, the enhancement is 15.8 . Thus, the resulting disk mass is rather insensitive to the assumed effective opacity, and we can estimate the total disk mass as $0.006 M_{\odot}$.

The mass of the $\mathrm{HH} 30$ circumstellar disk derived from $1.3 \mathrm{~mm}$ data is at the low end of the range for young stars associated with Herbig-Haro objects (Reipurth et al. 1993). It is typical of the mass associated with $T$ Tauri stars (Beckwith et al. 1990). This is consistent with the apparent lack of an extended infalling evelope and the view that $\mathrm{HH} 30$ may be nearing the end of its most active accretion phase.

At the mass accretion rates estimated for $\mathrm{HH} 30$, the disk mass will be completely consumed on a timescale of $10^{5} \mathrm{yr}$,

TABLE 4

Measured Optical Properties of Grain and Central Source

\begin{tabular}{cccccc}
\hline \hline Bandpass & $\langle\lambda\rangle_{B}$ & $g_{\lambda}=\langle\cos \theta\rangle$ & $A_{\lambda} / A_{\mathrm{F} 814 \mathrm{w}}$ & $(a / 0.5) L_{\lambda} / L_{\lambda_{\odot}}$ & $(a / 0.5) M_{\lambda}$ \\
\hline F814W (1995)...... & 7877 & 0.64 & 1.00 & 0.162 & $7.58 \pm 0.12$ \\
F675W (1995)...... & 6700 & 0.70 & 1.10 & 0.088 & $7.95 \pm 0.13$ \\
F555W (1994)...... & 5398 & 0.86 & 1.57 & 0.039 & $8.32 \pm 0.11$ \\
\hline
\end{tabular}


which is comparable to the system's age. Thus, HH 30 is probably at least halfway through its active accretion phase. This timescale is much larger than the outflow lifetime of $10^{3}$ yr obtained by dividing the jet extent by the observed proper motions. The average accretion rate must have been at least an order of magnitude higher during the first half of the accretion phase to supply a total mass above the hydrogen-burning limit. Therefore, we observe the $\mathrm{HH} 30$ accretion disk after it has processed most of the material onto the protostar. This conclusion is reinforced by the apparent lack of an extended envelope.

The age and diameter of the $\mathrm{HH} 30$ accretion disk compare well to models of the early solar nebula during or prior to planet formation. The total disk mass is about the mass of Jupiter: an order of magnitude smaller than the "minimum mass" solar nebula. Assuming that planetisimals or massive protoplanets have already formed within the shielded nebula, it appears that the HH 30 system could evolve a planetary system as massive as our own own.

\section{CONCLUSIONS}

HH 30 appears to be a young stellar object with an edge-on optically thick circumstellar disk. The star is not seen directly - only nebulosity is seen. We resolve $\mathrm{HH} 30$ into a highly collimated emission-line jet, a bright reflection nebula, a faint counternebula, and a faint counterjet. The two reflection nebulosities are caused by the central protostar illuminating the upper and lower surfaces of the flared and optically thick disk. They extend to an outer radius of about $250 \mathrm{AU}$.

The ionized jet is highly collimated and unresolved (less than 20 AU FWHM) 70 AU from its source. This is much narrower than the size of the cleared region revealed by the optical reflection nebula, and thus the jet collimation must occur very close to the star. The jet contains knots that have a significant range of proper motions between 100 and 300 $\mathrm{km} \mathrm{s}^{-1}$. The jet has an opening angle of $3^{\circ}$ within $5^{\prime \prime}$ of the source. The counterjet has a wider opening angle.

The brighter north-northeast reflection nebula decreased in brightness by $0.5 \mathrm{mag}$ in the year between the two observations, while the south-southwest nebula increased by about the same amount. A change in accretion luminosity on the two sides of the inner accretion disk, which also originates new jet knots asymmetrically every few years or so, could explain this.

We have pursued both single-scattering and multiplescattering models for the reflection nebulae. Singlescattering models run quickly, and we have done least-squares fitting to the image. At these solution points we have then calculated grids of multiple-scattering models. We have fitted this image successfully with a flared, selfconsistent optically thick disk model and have the following conclusions :

1. A disk inclination of less than $10^{\circ}$ is needed to match the brightness ratio of the reflection nebulosities.

2. The distribution of the scattered light is consistent with a pressure-supported disk, at least in the scattering region. The disk does not have an exponential profile, so attraction to the central object rather than the disk's selfgravity is significant in counteracting the pressure.

3. The material in the disk has a thermal time constant much less than the orbital or sound crossing time. This implies that the shielded regions of the disk are colder and, assuming pressure support, denser than an extrapolation from the observed scattering regions.

4. There is a degeneracy between the two parameters in the density distribution $\alpha$ (the radial volume density powerlaw index) and $\beta$ (the radial disk height power-law index). Larger $\beta$ can be counteracted by smaller $\alpha$. However, if the disk is pressure supported and in thermal equilibrium with the central protostar, this degeneracy is broken, and a unique solution is obtained if the (extrapolated) extinction to the protostar is known. In this case, the vertical structure of the disk depends on radius as $z(r) \sim r^{1.45 \pm 0.05}$. The primary uncertainty is the (extrapolated) extinction to the protostar, which lies between 10 and about $60 \mathrm{mag}$. If the contribution from the denser shielded region of the disk is included, the (extrapolated) extinction in the reference model (which is constrained to $30 \mathrm{mag}$ ) is increased by about 35 to $65 \mathrm{mag}$. The observational constraint is that the extinction should exceed $24 \mathrm{mag}$.

5. We derive a surface density profile that falls roughly as $1 / r$ : there is a roughly constant mass at each distance from the star. The effect of the shielding is to flatten the density profile.

6. Much better model fits are obtained if the HenyeyGreenstein phase function asymmetry parameter $g$ is increased beyond the nominal MRN ISM value of 0.3 at 0.8 $\mu \mathrm{m}$ wavelength. We find $g=0.64 \pm 0.06$, consistent with more recent determinations of the ISM value. We also are able to measure the standard interstellar extinction ratio for the $V$ and $I$ bandpasses, but the $R$ bandpass seems anomalous, perhaps due to emission-line contamination.

7. The models constrain the scale height at $100 \mathrm{AU}$ to be about $15 \mathrm{AU}$. The requirement of pressure support relates this scale height to a temperature of about $34 \mathrm{~K}$ at $100 \mathrm{AU}$; if the mass of the central protostar is $0.67 \mathrm{AU}$, then the bolometric luminosity is $1 L_{\odot}$. The brightness of the central source is 0.16 times solar in the $I$ bandpass (assuming a scattering albedo of 0.5 ), but the $V-I$ color is almost 1 , and presumably much of the source luminosity is in the nearinfrared.

8. The disk mass couples directly to the width of the dark absorption lane. Masses greater than $10^{-3} M_{\odot}$ produce an absorption lane that is too wide for nearly all choices of the other model parameters. This disk mass needs corecting for cooler central regions in the disk, and we derive a total gas + dust mass of $0.006 M_{\odot}$. The crude estimate of the accretion rate indicates a lifetime of about $10^{5} \mathrm{yr}$ for this phase of its evolution, which is about 2 orders of magnitude higher than the jet's dynamical age.

Further progress on $\mathrm{HH} 30$ will require both new observational and theoretical input. Direct IR measurement of the extinction to the central object will constrain the models further, as will planned polarization measurements and higher signal-to-noise ratio and resolution images with the Planetary Camera. A full self-consistent simulation of the disk and its temperature distribution would be of great value in verifying and refining the models presented here. It is also important to compare more detailed models of the disk with the observed infrared spectrum of this object. HH 30 is potentially the best accretion disk model that we can study because we resolve its spatial structure well, it does not appear to have an extensive envelope, and its orientation avoids scattered light problems from the central 
object. The asymmetries that do exist need explaining; are they infalling envelope material or disk instabilities (perhaps caused by hotter regions expanding and then being heated more as they catch more energy from the central source)? If the latter, then perhaps a method of generating turbulence in the disk is available. The jet is also of great interest because it can be resolved clearly and evolve over a timescale of months. It will be fascinating to see if it does precess as the images suggest or if the bend in it are due to interaction with the external medium.
This work was conducted partially at the Space Telescope Science Institute, operated by AURA, and at the Jet Propulsion Laboratory, California Institute of Technology, under contracts with the National Aeronautics and Space Administration. K. R. S. acknowledges support from the Origins of Solar Systems Research Program, Code SL, NASA Headquarters. We would like to thank Steve Beckwith, Doug Lin, John Mathis, Lynn Matthews, Reinhard Mundt, Nino Panagia, Jim Pringle, and an anonymous referee for valuable discussions and suggestions.

\section{REFERENCES}

Beckwith, S. V. W., \& Birk, C. C. 1995, ApJ, 449, L59

Beckwith, S. V. W., Sargent, A. I., Chini, R. S., \& Guesten, R. 1990, AJ, 99 924

Burrows, C. J., ed. 1995, Wide Field and Planetary Camera 2 Instrument Handbook (Baltimore: STScI)

Burrows, C. J., et al. 1996, in preparation

Cardelli, J. A., Clayton, G. C., \& Mathis, A. S. 1989, ApJ, 345, 245

Cohen, M., \& Jones, B. F. 1987, ApJ, 321, 846

Cohen, M., \& Schmidt, G. D. 1981, AJ, 86, 1228

Cohen, M., \& Schwartz, R. D. 1987, ApJ, 316, 311

Draine, B. T., \& Lee, H. M. 1984, ApJ, 285, 89

Elias, J. H. 1978, ApJ, 224, 857

Graham, J. A., \& Hayer, M. H. 1990, PASP, 102, 972

Holtzman, J. A., Burrows, C. J., Casertano, S., Hester, J. J., Trauger, J. T. Watson, A. M., \& Worthey, G. 1995, PASP, 107, 1065

Kenyon, S. J., \& Hartmann, L. 1995, ApJS, 101, 117

Krist, J. E. 1995, in ASP Conf. Ser. Vol. 77, Astronomical Data Analysis Software and Systems IV, ed. R. A. Shaw, H. E. Payne, \& J. M. E. Hayes (San Francisco: ASP), 349

Lin, D. N. C., \& Papaloizou, J. 1985, in Protostars and Planets II, ed. D. C Black \& M. S. Matthews (Tucson: Univ. Arizona Press), 981

Lopez, R., Raga, A., Riera, A., Anglada, G., \& Estalella, R. 1995, MNRAS, 274, 19

Mathis, J. S., Rumpl, W., \& Nordsieck, K. H. 1977, ApJ, 217, 425

Mundt, R., Brugel, E. W., \& Buhrke, T. 1987, ApJ, 319, 275

Mundt, R., \& Fried, J. W. 1983, ApJ, 274, 83

Mundt, R., Ray, T. P., Buhrke, T., Raga, A. C., \& Solf, J. 1990, A\&A, 232

Reipurth, B., Chini, R., Krugel, E., Kreysa, E., \& Sievers, A. 1993, A\&A 273,221

Rodriguez, L. F. 1996, private communication

Roddier, F. 1996, private communication

Ruden, S. P., \& Pollack, J. B. 1991, ApJ, 375, 740

Stahler, S. W. 1983, ApJ, 274, 822

Stapelfeldt, K. R., et al. 1995, ApJ, 449, 888

Strom, K. M., Strom, S. E., Edwards, S., Cabrit, S., \& Skrutskie, M. F. 1989, AJ, 97, 1451

Vrba, F. J., Rydgren, A. E., \& Zak, D. S. 1985, AJ, 90, 2074

Watson, A. M. 1994, Ph.D thesis, Univ. Wisconsin, Madison

Weidenschilling, S. J., \& Cuzzi, J. N. 1993, in Protostars and Planets III, ed. E. H. Levy \& J. I. Lunine (Tucson: Univ. Arizona Press), 1031

White, R. L. 1979, ApJ, 229, 954

Whitney, B. A., \& Hartmann, L. 1992, ApJ, 395, 529

. 1993, ApJ, 402, 605

Witt, A. N., Oliveri, M. V., \& Schild, R. E. 1990, AJ, 99, 888 